\title{
OPEN Sensitivity to food and cocaine cues are independent traits in a large sample of heterogeneous stock rats
}

\author{
Christopher P. King ${ }^{1}$, Jordan A. Tripi ${ }^{1}$, Alesa R. Hughson ${ }^{2}$, Aidan P. Horvath ${ }^{2}$, \\ Alexander C. Lamparelli ${ }^{1}$, Katie L. Holl ${ }^{3}$, Apurva S. Chitre ${ }^{7}$, Oksana Polesskaya ${ }^{7}$, \\ Keita Ishiwari ${ }^{4,5}$, Leah C. Solberg Woods ${ }^{6}$, Abraham A. Palmer ${ }^{7,8}$, Terry E. Robinson ${ }^{2}$, \\ Shelly B. Flage $\left.\right|^{9,10} \&$ Paul J. Meyer ${ }^{1 \bowtie}$
}

Sensitivity to cocaine and its associated stimuli ("cues") are important factors in the development and maintenance of addiction. Rodent studies suggest that this sensitivity is related, in part, to the propensity to attribute incentive salience to food cues, which, in turn, contributes to the maintenance of cocaine self-administration, and cue-induced relapse of drug-seeking. Whereas each of these traits has established links to drug use, the relatedness between the individual traits themselves has not been well characterized in preclinical models. To this end, the propensity to attribute incentive salience to a food cue was first assessed in two distinct cohorts of 2716 outbred heterogeneous stock rats (HS; formerly N:NIH). We then determined whether each cohort was associated with performance in one of two paradigms (cocaine conditioned cue preference and cocaine contextual conditioning). These measure the unconditioned locomotor effects of cocaine, as well as conditioned approach and the locomotor response to a cocaine-paired floor or context. There was large individual variability and sex differences among all traits, but they were largely independent of one another in both males and females. These findings suggest that these traits may contribute to drug-use via independent underlying neuropsychological processes.

Complex interactions between a host of genetic and environmental factors are thought to result in a number of intermediate traits that may confer vulnerability to develop impulse control disorders, including addiction. There has been considerable preclinical research on traits that predict drug self-administration behavior and susceptibility to relapse in order to better understand the neuropsychological bases of these vulnerability factors. For example, in rodents, behavioral phenotypes thought to influence drug-taking and -seeking behavior include the propensity to attribute incentive value to reward cues ${ }^{1-3}$, novelty-seeking ${ }^{4-6}$, locomotor response to novelty ${ }^{7-9}$, and impulsivity ${ }^{10-13}$.

Of these traits, we have been especially interested in how individual variation in the propensity to attribute incentive salience to rewards and their associated stimuli ("cues") influence the development of addiction-like behavior. When delivery of a food reward is paired with presentation of a cue (conditioned stimulus, CS; usually a lever) some rats (sign-trackers, ST) come to approach and interact with the CS itself ${ }^{14,15}$, whereas during the CS period others (goal-trackers, GT) approach and interact with the food cup ${ }^{15,16}$. These phenotypic differences predict a number of addiction-related behaviors ${ }^{17}$, including responses to drug and drug cues ${ }^{3,18}$, the ability of drug cues to support drug-taking behavior ${ }^{19}$, and the ability of drug cues to motivate drug-seeking behavior ${ }^{1,2,20}$. Sign-tracking is also associated with other traits thought to confer vulnerability of addiction, most notably, impulsivity and poor top-down attentional control over behavior ${ }^{21-23}$. However, the extent to which sign-tracking is associated with other unconditioned or conditioned drug responses is not well understood.

${ }^{1}$ Behavioral Neuroscience Program, Department of Psychology, University At Buffalo, Park Hall B72, Buffalo, NY 14260, USA. 'Department of Psychology, University of Michigan, Ann Arbor, USA. ${ }^{3}$ Department of Pediatrics, Human and Molecular Genetics Center and Children's Research Institute, Medical College of Wisconsin, Milwaukee, USA. ${ }^{4}$ Clinical and Research Institute On Addictions, Buffalo, USA. ${ }^{5}$ Department of Pharmacology and Toxicology, University At Buffalo, Buffalo, USA. ${ }^{6}$ Department of Internal Medicine, Molecular Medicine, Center on Diabetes, Obesity and Metabolism, Wake Forest School of Medicine, Winston-Salem, USA. ${ }^{7}$ Department of Psychiatry, University of California San Diego, La Jolla, USA. ${ }^{8}$ Institute for Genomic Medicine, University of California San Diego, La Jolla, USA. ${ }^{9}$ Department of Psychiatry, University of Michigan, Ann Arbor, USA. ${ }^{10}$ Michigan Neuroscience Institute, University of Michigan, Ann Arbor, USA. ${ }^{\circledR}$ email: pmeyer@buffalo.edu 


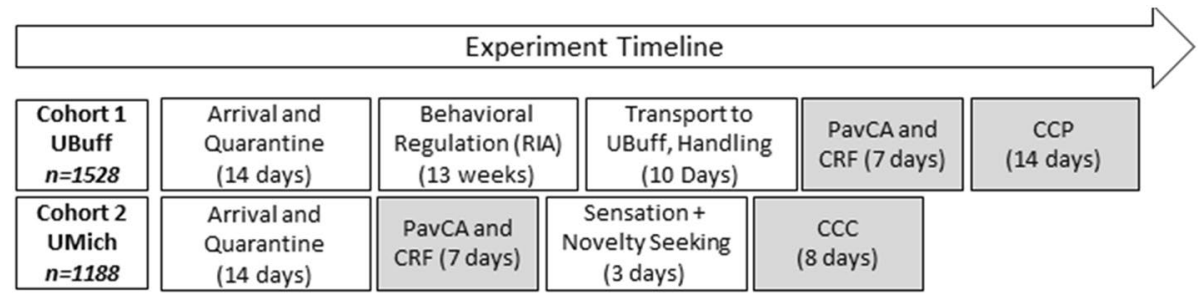

Table 1. Timeline for the University at Buffalo and Michigan cohorts. Rats arrived at both testing sites, and were quarantined for 14 days before entering the study. Note that the UBuff cohort was tested at the Research Institute on Addictions on several non-drug behavioral regulation tasks before being transported to the University at Buffalo. Rats were handled for a minimum of 7 days upon arrival. Blocks shaded in gray reflect the data presented in this paper.

We have begun to address sign-tracking in its relation to other traits as part of an ongoing genome-wide association study (GWAS). We report here the initial results from a sample of 2716 outbred heterogeneous stock (HS) (known also as NMcwi:HS; formerly known as N:NIH; N:NIH-HS) rats in two cohorts, which was established at the $\mathrm{NIH}^{24,25}$ from a cross of 8 inbred founder strains now maintained as a unitary outbred population. More information on this population is available at (https://ratgenes.org/cores/core-b/) (also described in: ${ }^{26}$ ). The propensity to approach a food cue was measured in all HS rats using the Pavlovian conditioned approach (PavCA) procedure; we then determined the extent to which this was correlated with (1) The unconditioned immediate locomotor activating effects of cocaine $\mathrm{e}^{27},(2)$ the conditioned approach response to a cocaine-paired floor stimulus ${ }^{28}$, and (3) the conditioned locomotor response to a cocaine-paired context ${ }^{29}$. We also assessed whether any of these effects were sex-dependent.

\section{Results}

The tendency to attribute incentive salience to a food-CS was assessed in two cohorts of HS rats using an identical PavCA procedure, one at the University at Buffalo (UBuff; $n=1528$ ) and another at the University of Michigan (UMich; $\mathrm{n}=1188$ ). We did not make direct statistical comparisons between the two cohorts because the UBuff cohort of rats underwent behavioral testing prior to PavCA, and thus the age of rats varied between the two cohorts (see Table 1). Both sites then examined the reinforcing properties of the lever-CS during a conditioned reinforcement $(\mathrm{CRF})$ procedure before separately measuring each cohort for either conditioned approach to towards a cocaine-paired floor, or conditioned locomotion in a cocaine-paired context. Here, we first present the PavCA data from the UBuff cohort replicating the findings of the UMich cohort (Figs. 1,2) before examining the relationship with unconditioned and conditioned cocaine responses. The UMich cohort of rats have been described in detail previously ${ }^{30}$.

Pavlovian conditioned approach (UBuff). During conditioning, rats learned to approach both the lever [main effect of Session: $F(4,6088)=1026.2 ; p<0.001$ ], and the food cup [main effect of Session: $F(4,6088)=44.3$, $p<0.001]$ during the 8 -s lever-CS period. To quantify individual differences in tendency to goal- and signtrack, the PavCA index was computed yielding a value ranging from -1 (goal-tracking) to 1 (sign-tracking), reflecting the overall tendency to approach the lever or food cup, as described previously: ${ }^{15}$. Relative to males, females showed a greater tendency to sign-track as reflected by a higher terminal index score [Session $\times$ Sex: $\mathrm{F}(4,6088)=11.3, p<0.001$ ] (Fig. 1a,b). This is consistent with the findings from the UMich cohort ${ }^{30}$. Despite the observed group sex difference, there was considerable individual variability, with a substantial number of signand goal-trackers, as well as intermediates across both sexes (Fig. 1c).

Box and Jitter Plots Because of the large sample size, we have opted to present the data shown in Fig. 1c and elsewhere as box plots (Fig. 1b). The notches reflect the $95 \%$ confidence interval for the distribution, where the center of the notch reflects the median. The colored regions of the box plot reflect the inner quartile range, while the remaining outer range of the plot reflects the outer quartile range. The vertical white line with hair ticks reflects the standard error of the mean (SEM), with the center of the line reflecting the location of the mean. Individual subject data is shown behind box plots as a jitter plot. All other box plots presented in this paper follow the same rules for plotting as Fig. 1b.

Figure 2 shows the time course of acquisition of lever- and food cup-directed responses in intermediates, STs and GTs, during the 5 sessions of PavCA training. As expected, sign-trackers showed higher probability of interaction with the lever [main effect of PavCAPheno: $F(8,6088)=447.6,(p<0.001)$ ] (Fig. 2a), a larger number of lever deflections [main effect of PavCAPheno: $F(8,6088)=537.7,(p<0.001)$ ] (Fig. 2b), and a shorter latency to deflect the lever [main effect of PavCAPheno: $F(8,6088)=447.6,(p<0.001)$ ] (Fig. $2 c)$, compared to goaltrackers. Similarly, goal-trackers showed a higher probability of entering the food-cup [main effect of PavCAPheno: $F(8,6088)=502.9,(p<0.001)$ ] (Fig. $2 \mathrm{~d}$ ), a larger number of food-cup entries [main effect of PavCAPheno: $F(8,6088)=428.6,(p<0.001)]$ (Fig. $2 \mathrm{e})$, and a shorter latency to enter the food cup [main effect of PavCAPheno: $F(8,6088)=480.3,(p<0.001)]$ (Fig. 2f), compared to sign-trackers. Thus, the ST/GT/IN phenotype distinction was robust across multiple PavCA behaviors. All six of these measures interacted with sex [Session $\times$ PavCAPheno $\times$ Sex interactions: $F \mathrm{~s}(8,6088):=19.9,8.4,19.5,2.5,4.1,3.5,(p s<0.01)]$, such that female sign-trackers and intermediates showed more lever deflections, quicker latency, and higher probability of lever contact than male 

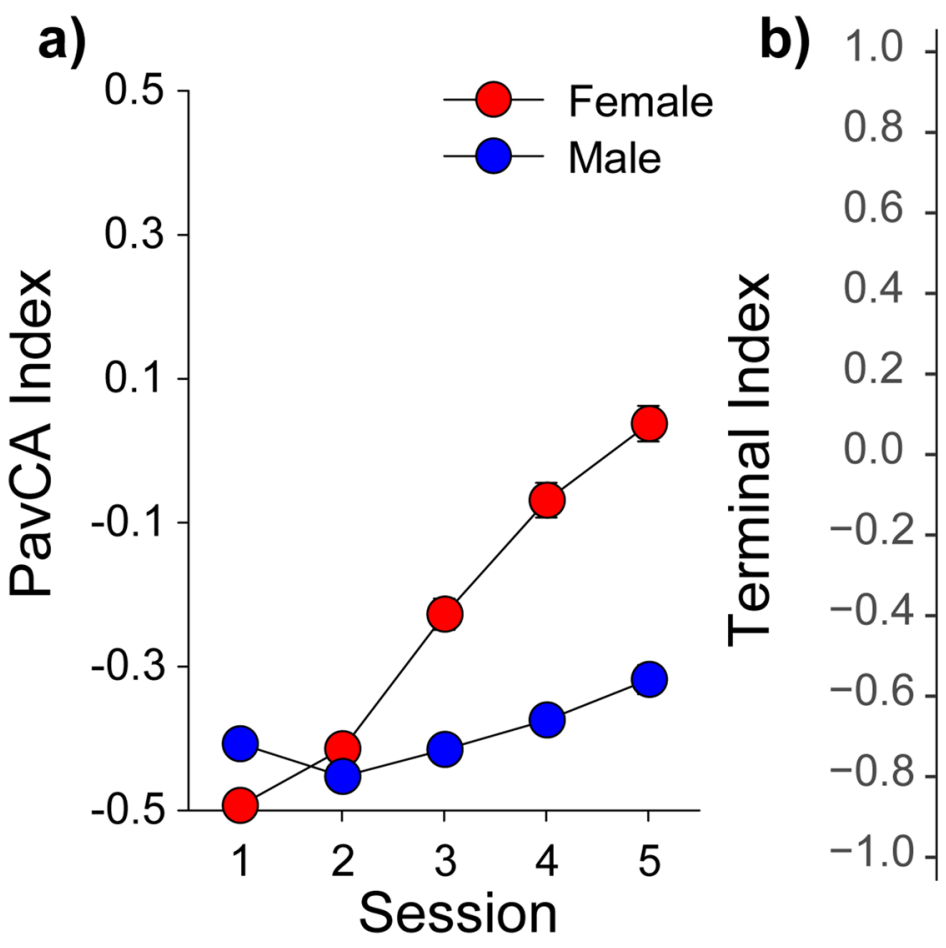

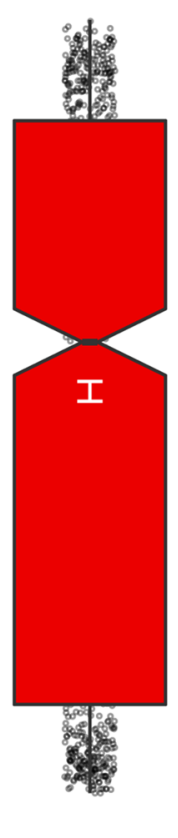

Females

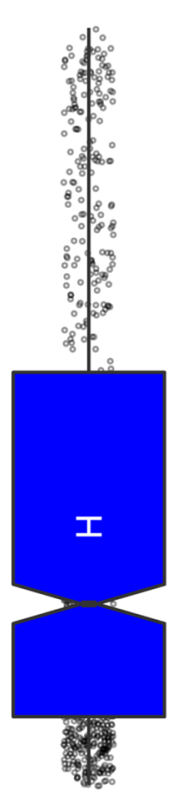

Males

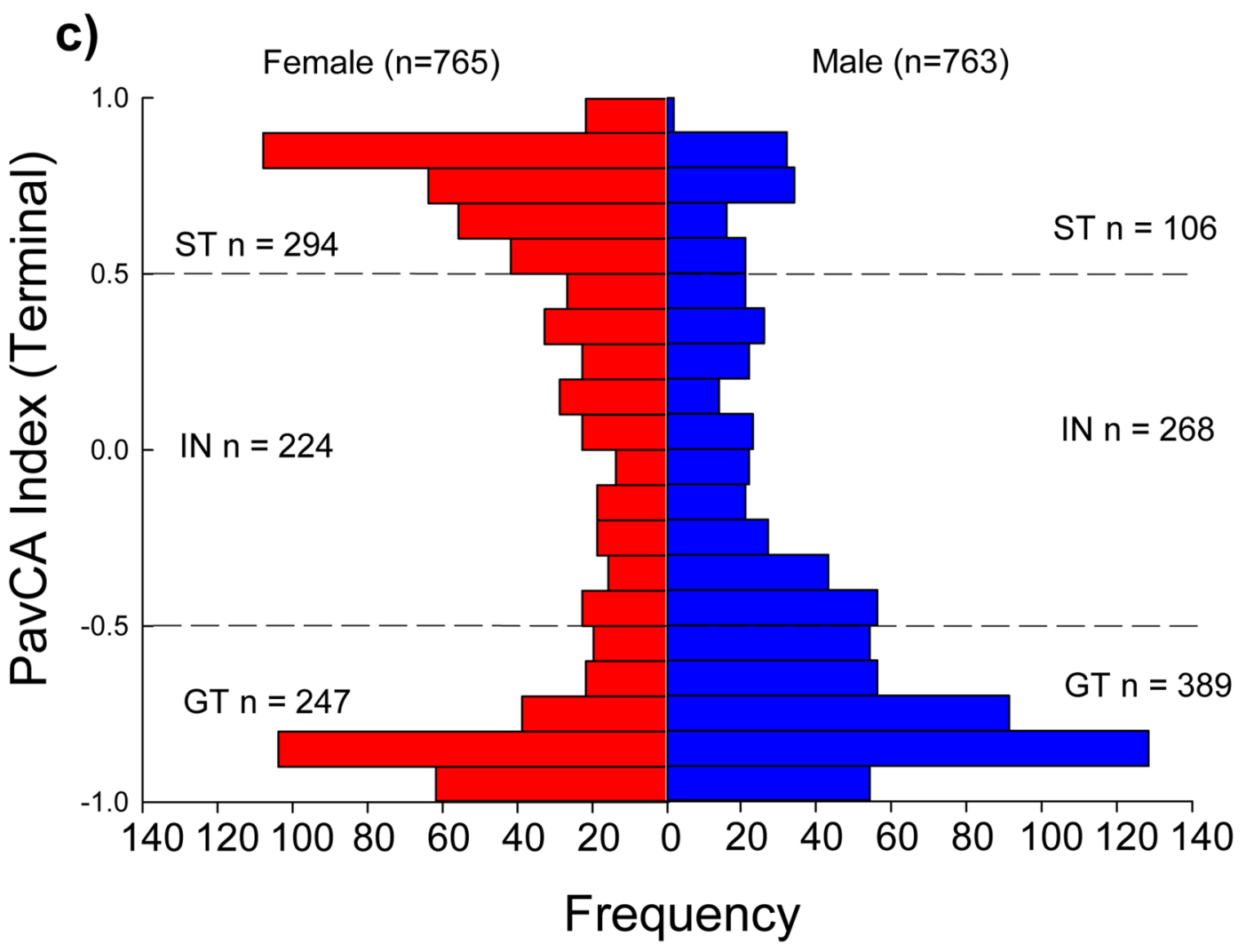

Figure 1. Sex differences during Pavlovian conditioned approach (PavCA) for the University at Buffalo Cohort (UBuff). Similar to the University of Michigan Cohort (UMich), across 5 sessions (total $n=1528$ ), (a) female rats were more likely to sign-track compared to males. However, despite this group difference, at the end of conditioning there was substantial heterogeneity in each group (c), with both males and females showing a large number of intermediates, sign- and goal-trackers. (b) The distribution of subjects across the possible values for index is shown as a box plot. See text for a description of statistics described by the box plots. 
Lever-Directed Behavior
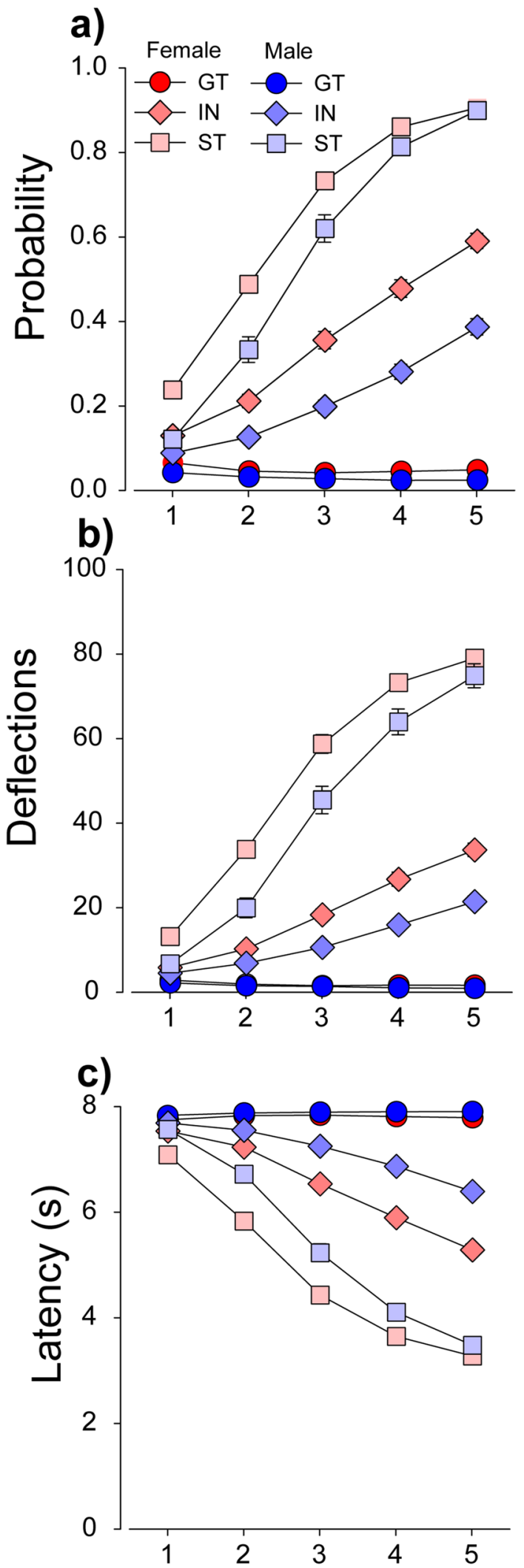

Food Cup-Directed Behavior

d)

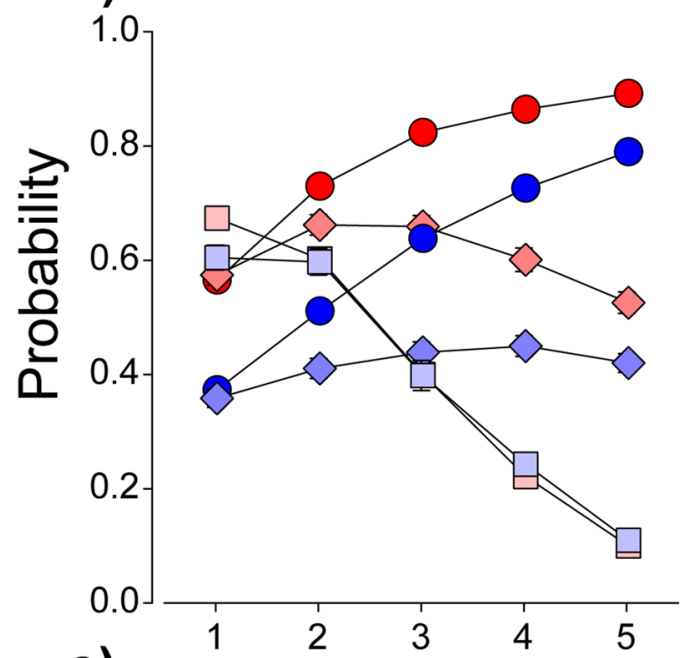

e)
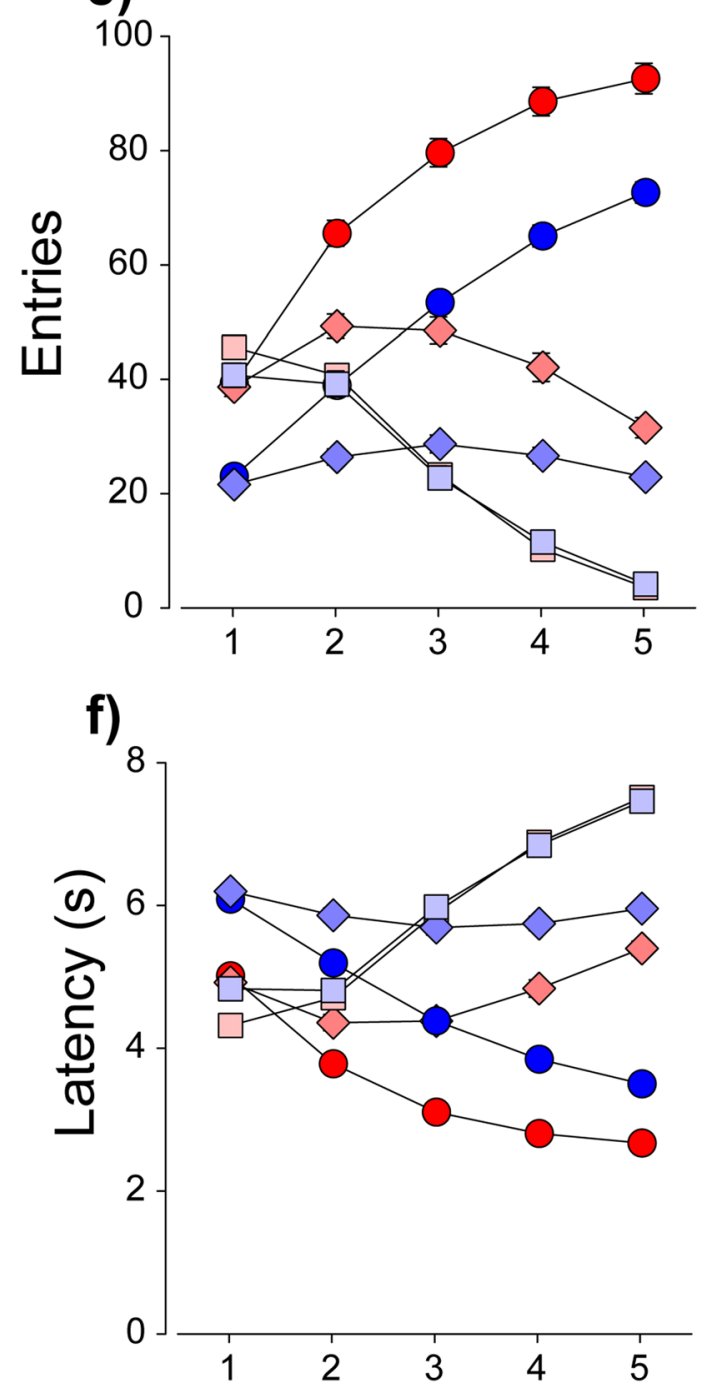

Figure 2. Individual measures of tendency to sign- and goal-track used to calculate PavCA index (UBuff). Performance of intermediates (IN), sign- (ST) and goal-trackers (GT) across 6 major behavioral measures during Pavlovian conditioned approach. Sign trackers showed (a) a higher probability of deflecting the lever, (b) increased number of lever deflections, and (c) faster latency to deflect the lever than intermediates and goal-trackers. Further, this effect was larger in females across all three PavCA phenotypes. Conversely, goal-trackers showed (d) higher probability of entering the food cup, (e) more food cup entries, and (f) quicker latency to enter the food cup than intermediates and sign-trackers. Similarly, this effect was more robust in females across all three measures. 
sign-trackers and intermediates (Fig. 2). Similarly, female goal-trackers and intermediates showed more food-cup entries, quicker latency, and higher probability of food cup entry than males did (Fig. 2). During the intertrial interval between lever presentations, females also showed a higher tendency to engage the food-cup across all 5 sessions [Sex $\times$ Session interaction: $F(4,5980)=76.2, p<0.001$ ] (data not shown), suggesting increased general activity in females relative to males. Again, these results are very similar to those described in the UMich cohort ${ }^{30}$.

Conditioned reinforcement. Next, rats were tested for the conditioned reinforcing properties of the lever stimulus using a Conditioned Reinforcement (CRF) test, in which rats learned to nosepoke for presentations of the lever-CS, as described previously ${ }^{31}$. During conditioned reinforcement, three measures of the conditioned reinforcing effect of the lever were measured: (1) active-directed responses, (2) number of earned lever presentations, and (3) number of lever deflections per presentation.

The lever served as an effective reinforcer in all rats [main effect of Port: $F(1,1522)=1718.0,(p<0.001)]$, although it was a more effective conditioned reinforcer in sign-trackers than goal-trackers and intermediates for all three measures: active responding [Port $\times$ PavCAPheno: $F(2,1522)=93.7,(p<0.001)$ ] (Fig. 3a, shown as active-inactive), earned reinforcers [main effect of PavCAPheno: $F(2,1522)=196.4,(p<0.001)$ ] (Fig. 3b), and lever presses per reinforcer [main effect of PavCAPheno: $F(2,1510)=83.4,(p<0.001)$ ] (Fig. $3 c)$. Further, this effect was also stronger in females for both earned reinforcers [PavCAPheno $\times$ Sex interaction: $F(2,1522)=3.5$, $(p<0.05)$ ] and lever deflections per reinforcer [PavCAPheno $\times$ Sex interaction: $F(2,1510)=6.3,(p<0.01)$ ] . Indeed, variance in PavCA accounted for 38 and $48 \%$ of the variance in lever presses per reinforcer in males and females, respectively $(p<0.05)$ (Fig. 3f). This particular measure most directly reflects the incentive value of the lever during this task; in comparison the other two measures, while also significantly correlated, accounted for much less of the variability between PavCA and CRF (Fig. 3d,e). These results are also consistent with those reported in the UMich cohort ${ }^{30}$, and further support the notion that the lever-CS was attributed with greater incentive salience in sign-trackers than goal-trackers ${ }^{31}$.

Cocaine cue preference: locomotion. Next, we observed that the relationship between the propensity to attribute incentive salience to a food CS, and the unconditioned locomotor effects of cocaine were independent the UBuff cohort. Specifically, a cocaine conditioned cue preference (CCP) procedure was used, whereby locomotor activation following a $10 \mathrm{mg} / \mathrm{kg}$ i.p. injection of cocaine was measured over four conditioning trials. Importantly, each trial consisted of a single injection of cocaine and saline, in alternation, on a discrete cocaine or saline paired floor stimulus. Cocaine induced significant locomotor activation compared to saline across all 4 conditioning trials [Drug $\times$ Trial interaction: $F(3,4572)=14.9,(p<0.001)$ ], which increased by trial 4 compared to trial 1, reflecting sensitization (Fig. $4 \mathrm{c})$. Further, the locomotor activating effect was larger in females than in males across each of the 4 sessions [Drug $\times$ Sex interaction: $F(1,1524)=419.9,(p<0.001)$ ] (Fig. $4 c)$. However, there was no significant effect of PavCAPheno on either Day 1 or Day 4 of testing ( $p s>0.05)$ (Fig. $4 \mathrm{a}, \mathrm{b})$, indicating that INs, STs and GTs did not differ in their locomotor response to cocaine. Indeed, there was no correlation between the PavCA Index score and cocaine-induced locomotor activity in either males or females (Fig. 4d). When all 4 sessions of conditioning were evaluated in a single analysis, there was a significant Drug $\times$ Trial $\times$ PavCAPheno interaction $[F(6,4572)=2.17,(p<0.05))]$, but the effect size was very small $\left(\eta^{2}=0.003\right)$. Further, although there was an effect PavCA phenotype on baseline locomotion during the habituation session [main effect of PavCAPheno: $F(2,1522)=10.2,(p<0.001)$ ] and on the first saline trial [main effect of PavCAPheno: $F(2,1522)=6.1,(p<0.001)]$, these effect sizes were also small $\left(\eta^{2}=0.013,0.007\right.$ respectively $)$. Thus, the tendency to sign- or goal-track is largely unrelated to the locomotor response of both acute and repeated injections of cocaine at the $10 \mathrm{mg} / \mathrm{kg}$ dose during CCP, and does not appear to be meaningfully related to locomotion under non-drug conditions.

Cocaine contextual conditioning: locomotion. Although a $10 \mathrm{mg} / \mathrm{kg}$ dose of cocaine is on the ascending limb of the cocaine locomotor dose response curve ${ }^{32}$, one of the hallmark features of sensitization to higher doses of cocaine is the development of stereotypy ${ }^{33}$ which include repetitive head movements (head waving) in rodents. Thus, in the UMich cohort of rats, a cocaine contextual conditioning (CCC) procedure was used to examine the development of both-cocaine induced locomotion and bouts of headwaving that reflect instances of stereotypy at the start and end of 5 daily conditioning trials to a $15 \mathrm{mg} / \mathrm{kg}$ dose of cocaine. In this case, headwaving bouts were computer recorded when the rat was not exhibiting locomotion, but in place and moving its head side-to-side, as previously described ${ }^{27}$.

Unlike CCP, here rats underwent testing in a constant context characterized by a wire-mesh floor and grey walls. Rats were allowed one session to habituate to the testing environment (day 1), followed by a baseline session (day 2), prior to which all rats received saline injections. On the first cocaine conditioning trial (day 3), cocaine acutely increased locomotor activity relative to baseline day 2 [main effect of Trial: $F(1,1167)=1926.2$, $(p<0.001)]$, and this effect was more robust in females [Trial $\times$ Sex: $F(1,1167)=63.4,(p<0.001)]$ (Fig. 5a). A three-way interaction [Trial $\times$ PavCAPheno $\times$ Sex: $F(2,1167)=3.1,(p<0.05)]$ revealed that on the first cocaine treated day (day 3 ), female STs showed a modest increase in locomotor activity compared to male STs and female GTs, although there were no phenotype differences within males (Fig. 5a). Cocaine also produced modest bouts of head waving on the first trial [main effect of Trial: $F(1,1167)=83.7,(p<0.001)$ ], and this effect was greater in females than males [Trial $\times$ Sex: $F(1,1167)=56.0,(p<0.001)]$, but was not affected by PavCAPheno (Fig. 5c).

We next examined the development of sensitization following five days of conditioning (day 3-7). Here, all subjects showed a decrease in locomotor activity by the end of conditioning [main effect of Trial: $F(1,1167)=276.6,(p<0.001)]$, and this decrease was more pronounced in females than males [Trial $\times$ Sex: $F(1,1167)=58.1,(p<0.001)]$. That is, females showed the greatest change in locomotor behavior from day 3 to 


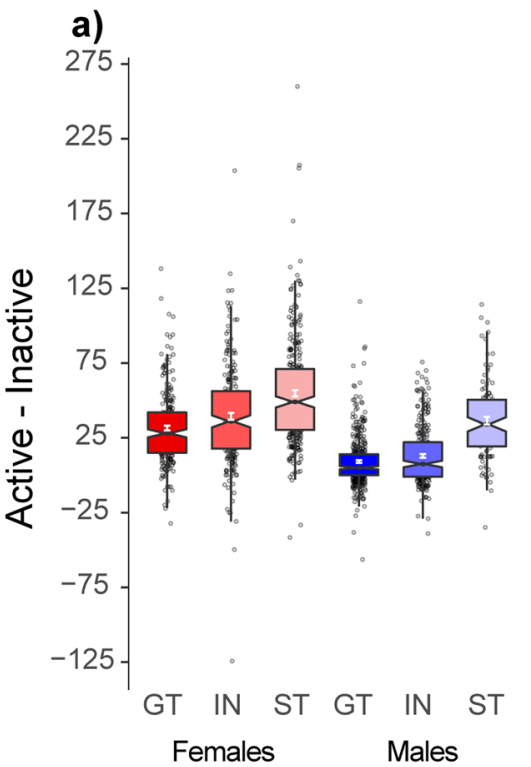

d)

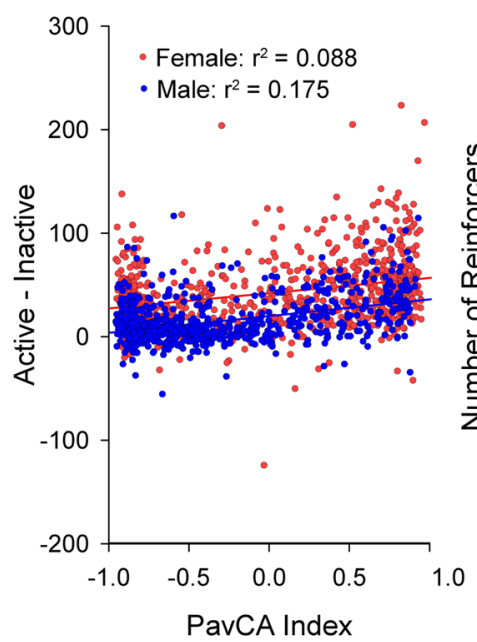

b)

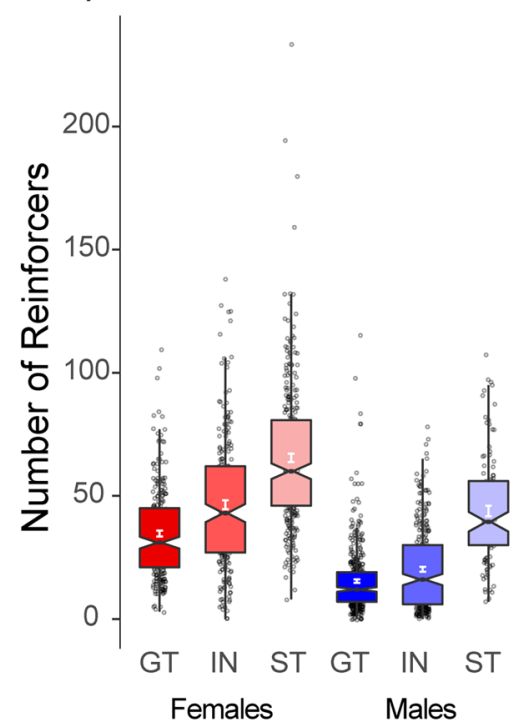

e)

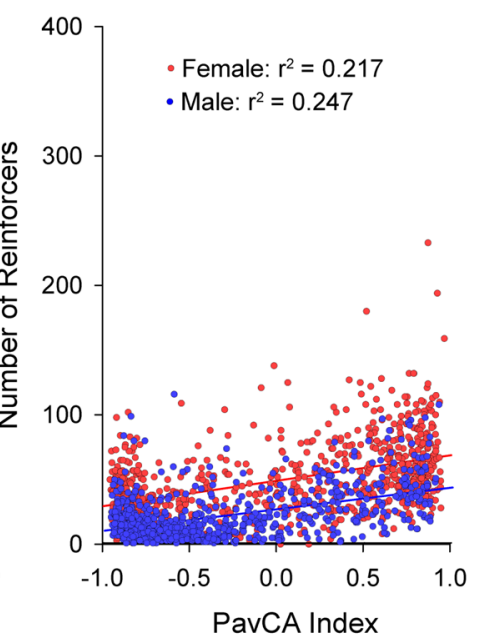

c)

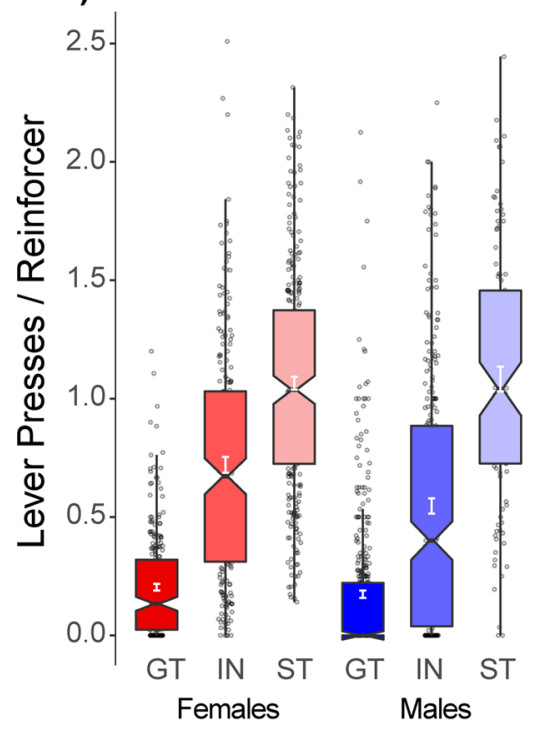

Figure 3. Conditioned Reinforcement (UBuff): Intermediates (IN), sign- (ST) and goal-trackers (GT) learned to nosepoke for 3-s presentations of the conditioned lever stimulus. On average, all phenotypes directed their responding to the active hole (a), although this effect was largest in sign-trackers. Consequently, (b) the number of times the subject was reinforced was also larger in sign-trackers than intermediates and goal-trackers. Further, (c) sign-trackers interacted with the lever stimulus the most during this test, followed by intermediates and goal-trackers. Across all subjects, PavCA index was correlated with (d) number of responses for the lever-CS, (e) earned lever-CS reinforcers, and (f) lever presses per reinforcer. The strongest correlation was between index and lever presses per reinforcer, presumably because number of lever deflections more directly reflect sign-tracking behavior.

day 7 (Fig. 5b). This decrease in locomotor activity was accompanied by a robust increase in head waving [main effect of Trial: $F(1,1167)=968.1,(p<0.001)$ ], and again this effect was larger in females than males [Trial $\times$ Sex: $F(1,1167)=145.2,(p<0.001)]$ (Fig. $5 d)$. There was however no effect of PavCAPheno following the 5 conditioning sessions, and tendency to sign- or goal-track appears largely unrelated to the unconditioned locomotor activating effects of cocaine.

Cocaine cue preference: conditioned approach. The tendency to sign-track is characterized by approach to reward-predictive cues. To test whether the tendency to sign-track was related to approach a discrete drug-paired cue, in this case a tactile floor, the change in preference for a cocaine-paired floor stimulus during CCP was examined. Subjects in the UBuff cohort were measured for "grid" and "hole" floor preference before and after one of these floors was paired with 4 injections of $10 \mathrm{mg} / \mathrm{kg}$ cocaine. Subjects were counterconditioned, such that cocaine was paired with the less preferred floor stimulus during the pre-test. Previously, Sprague-Dawley sign-trackers showed a robust conditioned cue preference to a cocaine-paired floor stimulus, 

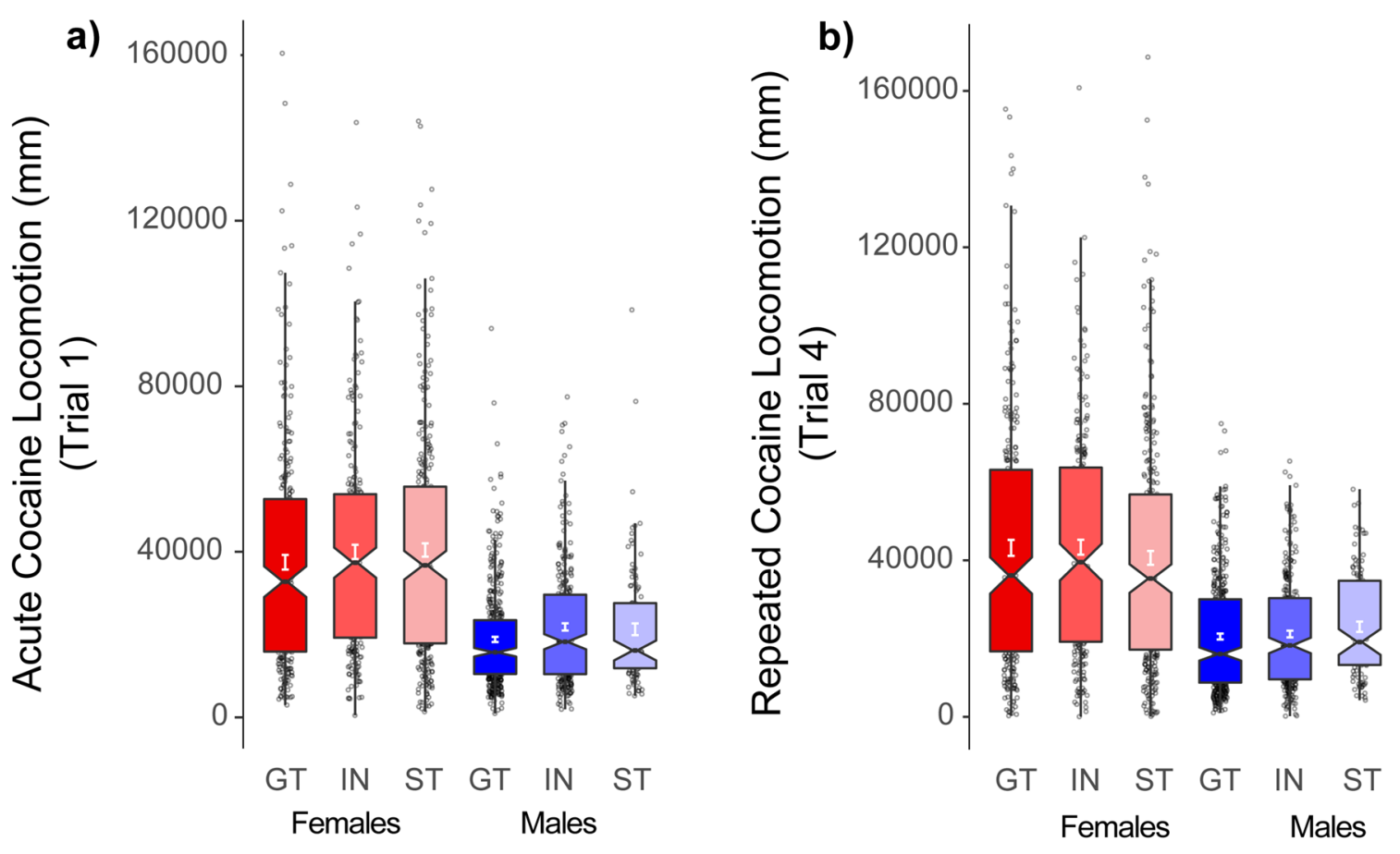

c)
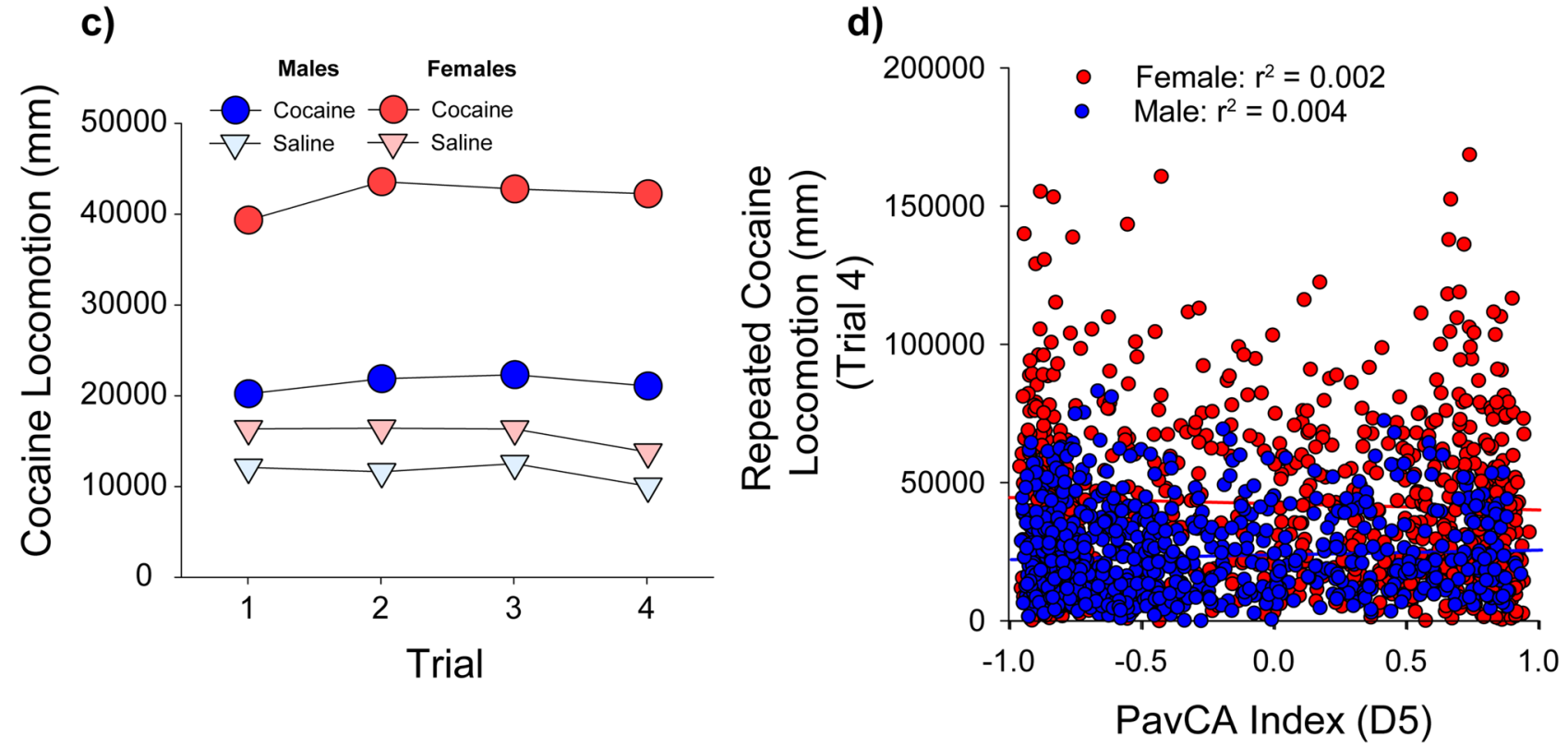

Figure 4. Unconditioned locomotor response during Conditioned Cue Preference (CCP) (UBuff): Rats showed robust locomotor activation to cocaine across 4 trails of conditioning. On the first trial (a) females showed larger cocaine-induced locomotion compared to males, although this effect was not different between intermediates (IN), sign- (ST), or goal-trackers (GT). (b) This sex difference persisted to the fourth trial of conditioning. (c) Cocaine induced larger locomotor activity compared to saline across trials, and this effect sensitized between sessions 1 and 4. (d) PavCA index is largely unrelated to cocaine induced locomotion at the end of conditioning in both males and females.

whereas goal-trackers did not ${ }^{3}$. Here, we sought to characterize this relationship using the population heterogeneity of HS rats, and although we expected that sign-trackers would show more robust expression of cue preference, here we observed these two traits were unrelated.

All groups showed a significant increase in preference for the cocaine paired floor following conditioning [main effect of Test: $F(1,1522)=1029.7,(p<0.001)$ ], and this effect was larger in females than males [Test $\times$ Sex: $F(1,1522)=8.22,(p<0.01)]$. Goal-trackers actually showed the greatest increase in preference for the cocaine paired floor [Trial $\times$ PavCAPheno: $F(2,1522)=4.83$, $(p<0.01)$ ] (Fig. 6a). However, this is likely due to the counterconditioning design used. During the pre-test, goal-trackers exhibited stronger bias against the cocaine paired floor prior to conditioning (data not shown). Further, PavCA index was uncorrelated with time spent on the 
a)

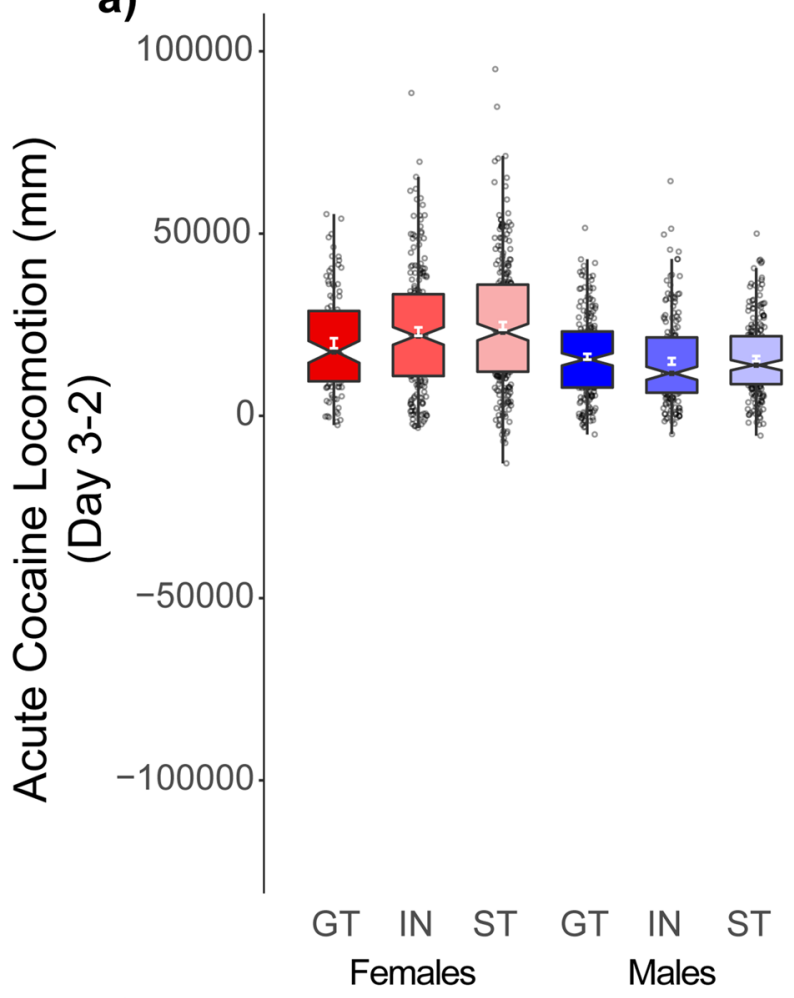

c)

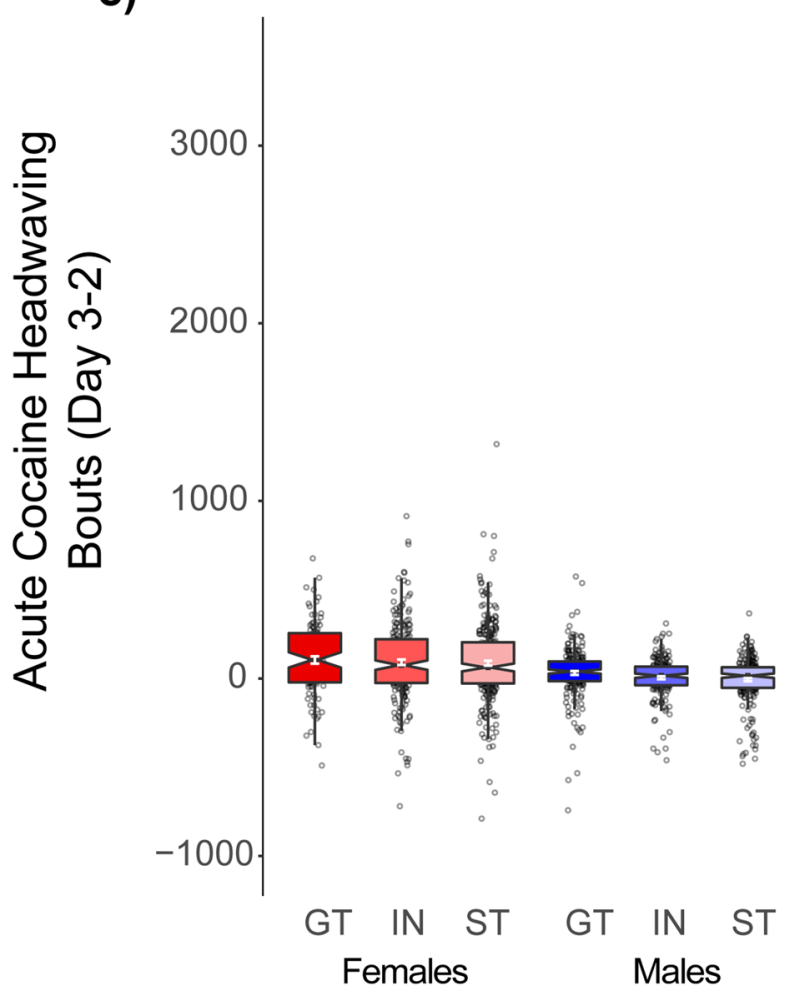

b)

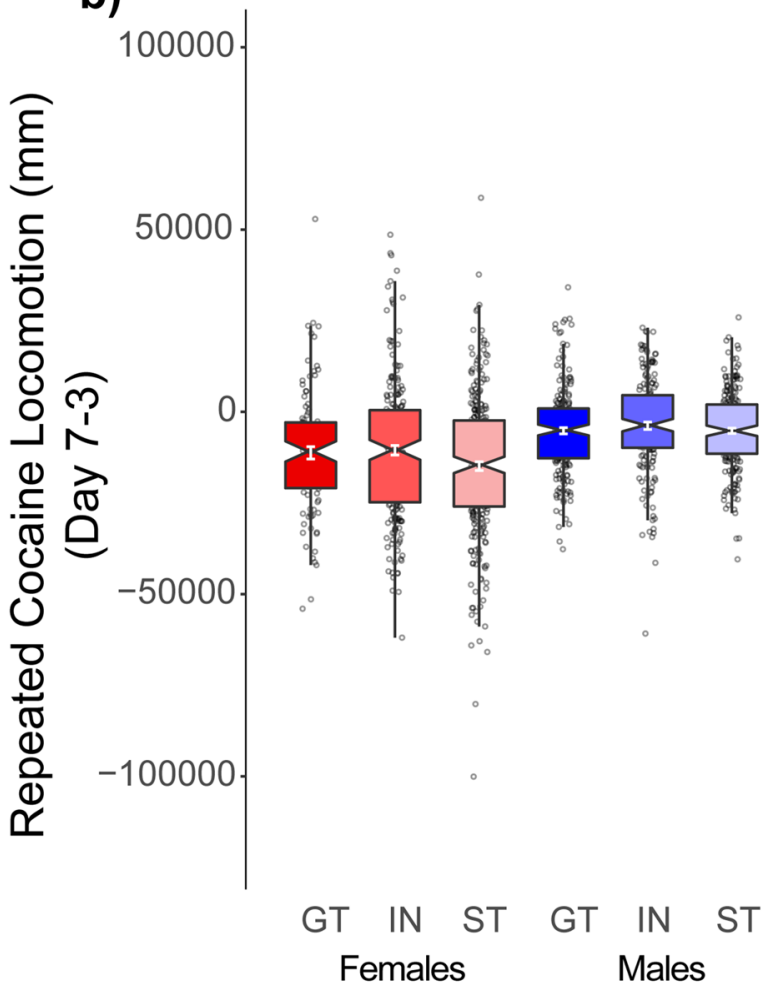

d)

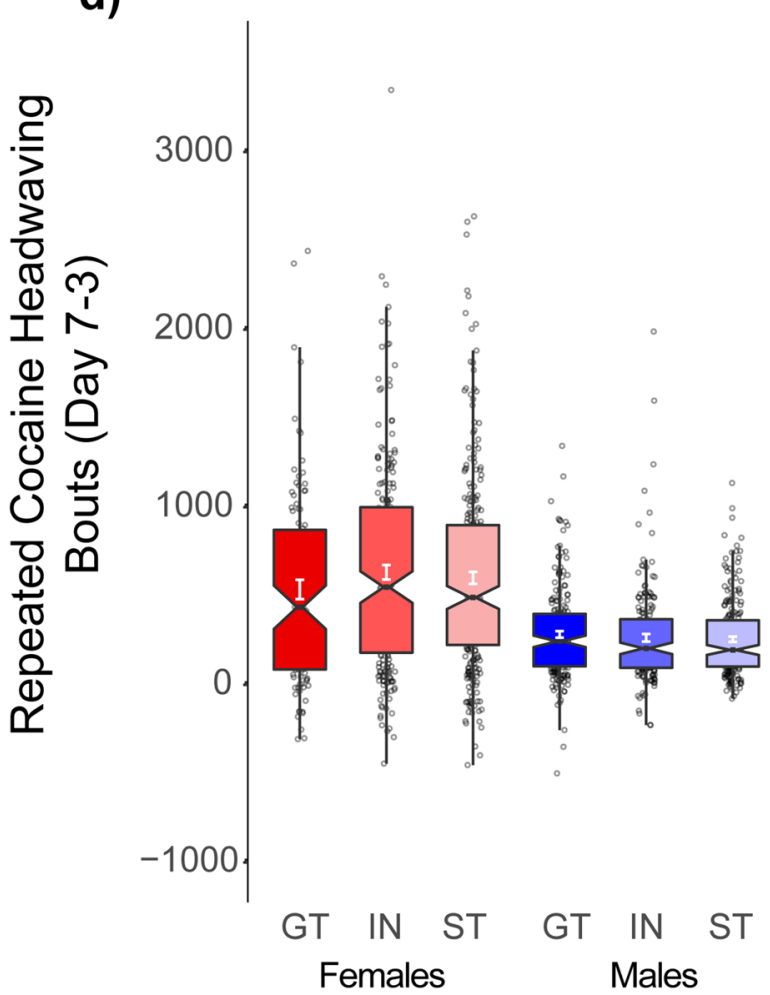

Figure 5. Unconditioned locomotor response during Cocaine Contextual Conditioning (CCC) for University of Michigan cohort (UMich): Rats showed (a) significant locomotor activation on the first session of conditioning compared to baseline, and this effect was larger in females than males. There were no differences between intermediates (IN), sign- (ST), and goal-trackers (GT). Further, (b) cocaine treatment induced headwaving on this first session as well, although neither locomotor activity nor headwaving was related to tendency to sign- or goal-track. On the final day of conditioning, subjects showed a (c) decrease in cocaine induced locomotion, and (d) an increase in cocaine-induced headwaving, and this effect was higher in females. Further, this effect was independent from PavCA phenotype. 

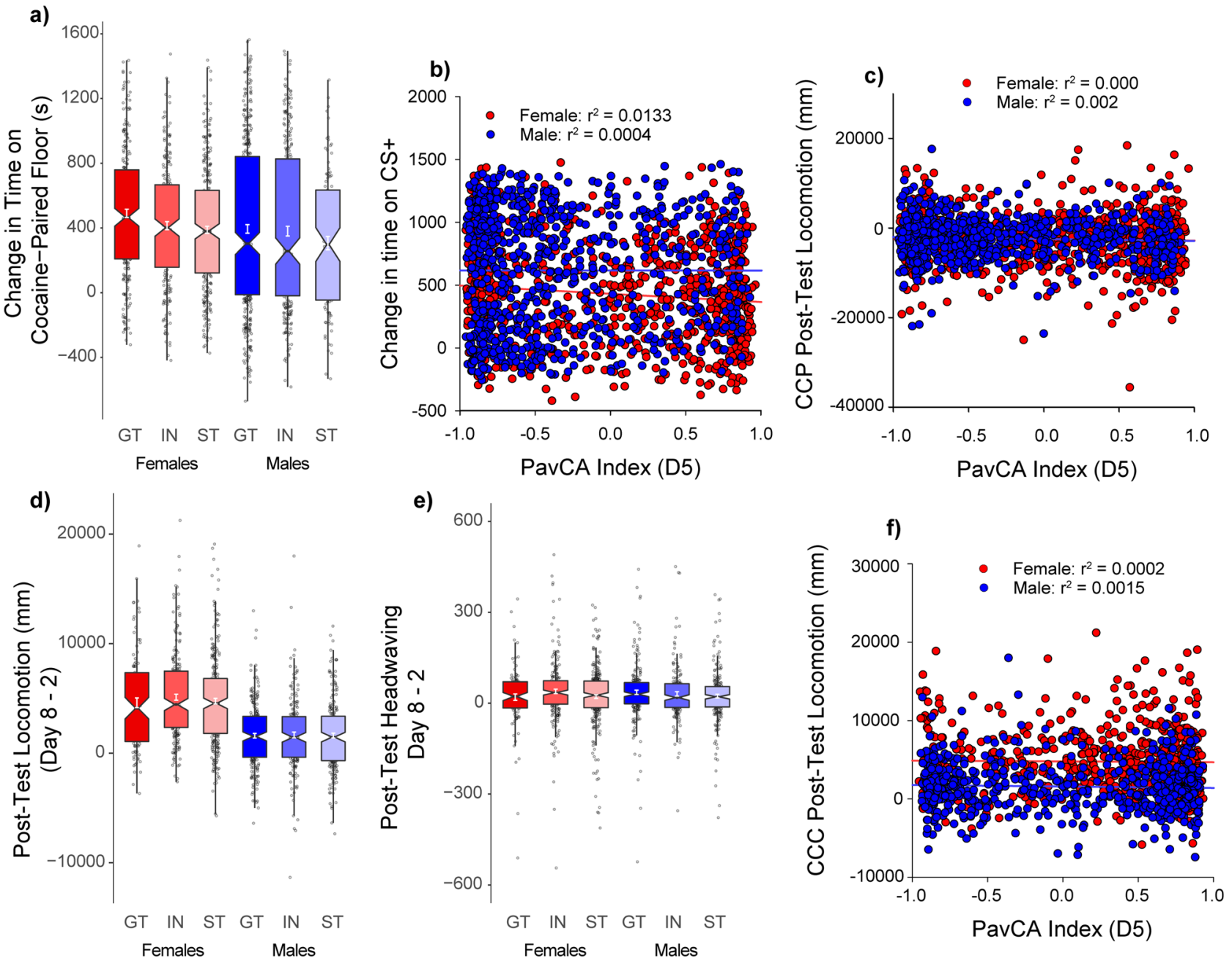

Figure 6. Conditioned approach and locomotion during Conditioned Cue Preference (CCP) and Cocaine Contextual Conditioning (CCC) (UBuff and UMich cohorts): During CCP following 4 saline and cocaine parings with two different tactile floor types, (a) subjects showed an increase in time spent on the cocaine paired floor following conditioning. However, (b) despite the heterogeneity in magnitude of conditioning, change in time spent on the cocaine paired floor showed no correlation with PavCA index. Further, (c) locomotor activity instigated on the post-test by the presence of the cocaine-paired floor was also unrelated to PavCA index. During CCC, (d) subjects showed increased conditioned locomotion on the post-test by the cocaine paired context, and this effect was larger in females. However, neither conditioned locomotion nor (e) conditioned headwaving were different between intermediates (IN) sign- (ST), or goal-trackers (GT). Hence, (f) no significant correlation was detected between index and conditioned locomotor activity.

cocaine-paired floor (Fig. 6b). Whether the PavCA index score was related to an increase in locomotor activity during the post-test relative to pre-test, was also examined, but these two measures were not significantly correlated (Fig. 6c). Thus, tendency to sign- or goal-track did not meaningfully inform the magnitude of conditioning for the cocaine-paired floor type.

Cocaine contextual conditioning: conditioned locomotion. We further examined the UMich cohort to determine whether a cocaine-paired context would elicit conditioned locomotion following conditioning, and whether this was related to behavior during PavCA. Unlike CCP where a tactile floor was the cocaine predictive stimulus, here the whole testing environment served as a cocaine-predictive context. Locomotor activity during an initial session prior to conditioning (day 2; prior to conditioning, but following saline injection) was compared to that on day 8 , which followed 5 sessions (day 3-7) of cocaine injections paired with that context conditioning (day 8 ). On day 8 , exposure to the cocaine-paired context elicited greater locomotor activity than that on day 2 [main effect of Test: $F(1,1167)=789.4,(p<0.001)$ ], and this effect was larger in females [Test $\times$ Sex: $F(1,1167)=194.5$, $(p<0.001)]$ (Fig. $6 \mathrm{~d})$. However, there was no effect of PavCAPheno on conditioned locomotion ( $p s>0.05)$. Similarly, although there was a modest increase in observed headwaving following conditioning [main effect of Test: $F(1,1167)=85.7,(p<0.001)$ ], this effect was minimal compared to conditioned locomotor activity (Fig. 6e) and was not related to Sex or PavCAPheno. PavCA index was not correlated with conditioned locomotion (Fig. 6f). These results indicate that attribution of incentive salience to reward cues, as measured by 

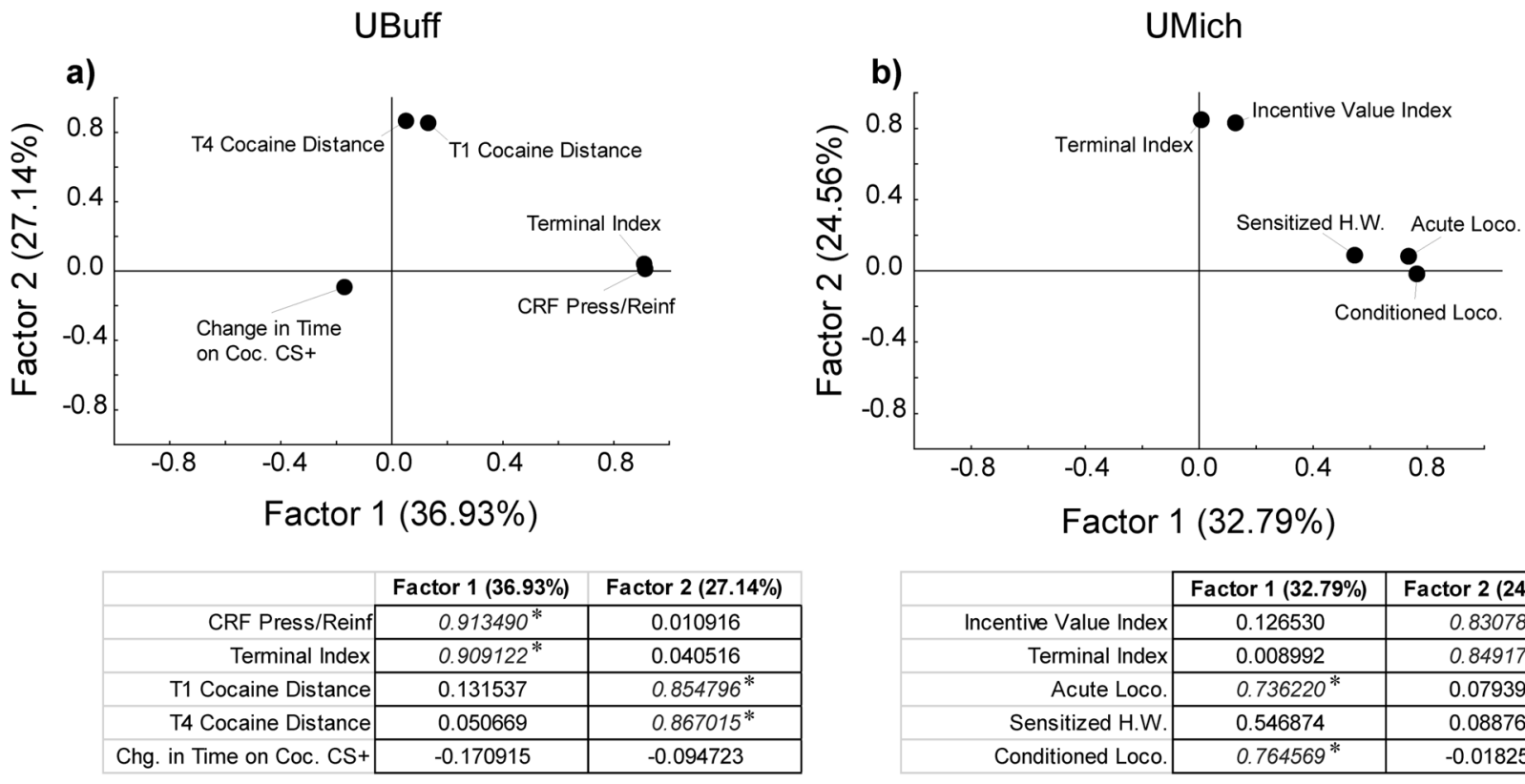

Figure 7. Principal components analysis of Pavlovian Conditioned Approach, Conditioned Cue Preference (CCP), and Cocaine Contextual Conditioning (CCC) (UBuff and UMich Cohorts). In both cohorts, we examined whether the traits examined could be reduced to more basic dimensions using principal components analysis. For both cohorts, two factors explained a majority of variance in these studies. In the Buffalo cohort (a) factor 1 showed significant loading from terminal index and interaction with the lever during conditioned reinforcement (CRF), while factor 2 showed significant loading from cocaine-induced locomotion during CCP. Similarly, in the Michigan cohort (b), factor 1 showed significant loading from the immediate (Acute Locomotion, Sensitized Headwaving) and conditioned locomotor (Conditioned Loco.) response to cocaine, whereas, factor 2 showed significant loading from terminal index and incentive value index during CRF. Individual factor loadings for included measures are shown below each panel. Asterisked values indicate factor loadings that exceed 0.7 .

approach to a food CS (ST), is independent of both approach to a cocaine-paired floor stimulus, and the conditioned locomotor response to a cocaine-paired context.

Principal components analysis. Principal components analyses were conducted to determine whether the relationship between conditioned and unconditioned responses to cocaine, and the propensity to attribute incentive salience to a food CS could be reduced to fewer dimensions. The measures included the primary measures from PavCA (Index and lever directed behavior during CRF), the acute and repeated unconditioned locomotor responses to cocaine, and conditioned approach and conditioned locomotion responses to cocainepaired stimuli. In each cohort, two major factors accounted for the majority of variance in these measures.

PCA for the UBuff PavCA-CCP cohort revealed that two factors accounted for $64 \%$ of the total variance. The first factor, which accounted for $36.9 \%$ of the variance, had strong loadings from both lever presses per reinforcer during CRF, and terminal PavCA index ( $>0.9)$, with non-significant loadings from CCP measures (Fig. 7a). Conversely, factor 2 had strong loadings from Trial 1 and Trial 4 cocaine induced locomotion $(>0.8)$, with non-significant loadings from change in time spent on cocaine-paired floor and PavCA measures (Fig. 7a). Together, this further supports the notion that PavCA, and the unconditioned and conditioned measures during CCP are independent.

A similar pattern of results was found for the UMich PavCA-CCC cohort, where two factors accounted for more than half the variance. The first factor accounted for $\sim 38 \%$ of the variance and contained strong loadings from both acute locomotion and the conditioned locomotor response to the cocaine-paired context $(>0.7)$ (Fig. 7b), with non-significant loadings from the PavCA measures and sensitization of the headwaving response. Factor 2, by comparison, had strong loadings ( $>0.8$ ) from both PavCA measures (Fig. 7b) and non-significant loadings from the CCC measures. This finding further suggests that, similar to CCP, behavior during CCC and PavCA are largely unrelated to each other.

\section{Discussion}

The purpose of the present study was to determine correlations between multiple addiction-related traits in a large sample of genetically diverse heterogeneous stock rats. The advantage of this approach is that it allows for multiple within-subjects comparisons across a broad range of behaviors and testing paradigms, each of which are thought to examine different but potentially related psychological processes. To this end, we used $n=2701$ rats from two separate cohorts to examine Pavlovian conditioned approach and its relationship to both conditioned and unconditioned responses to cocaine in two different tasks. Specifically, two cohorts of rats, one from UBuff 
and the second from UMich, were phenotyped for their tendency to engage a reward-predictive lever stimulus during the Pavlovian conditioned approach task. Next, performance during PavCA was compared to performance during $\mathrm{CRF}$, and to the locomotor activating effects of cocaine acutely and after repeated exposure during either a CCP or CCC task. Finally, PavCA and CRF was compared to the conditioned approach to a cocaine-paired floor, or conditioned locomotion to a cocaine-paired context during CCP and CCC, respectively.

Our findings were consistent with some previous reports but not others. First, the tendency to sign- or goaltrack was significantly correlated with performance during the conditioned reinforcement test ${ }^{15,21,30,31}$, in which rats were allowed to nosepoke for presentations of the Pavlovian lever-CS. Second, although there was substantial variability in locomotor activation during both CCP and CCC, PavCA did not correlate with locomotion during either task, consistent with others who have also reported the tendency to sign-track as having either a subtle or unrelated effect on locomotion to a novel environment ${ }^{34}$ or following cocaine treatment ${ }^{5,18,35}$. Indeed, unlike selectively-bred high-responder rats, who show increased locomotion to a novel environment and a greater tendency to sign-track relative to bred low-responders ${ }^{36,37}$, both locomotion and tendency to sign-track were independent in HS rats. Third, whereas food cues acquire incentive motivational properties to a much greater extent in STs than GTs, our hypothesis that STs would prefer a cocaine-associated floor cue (based on ${ }^{3}$ ) was not supported. Finally, the conditioned locomotor effects during CCC were not related to PavCA. Thus, there were no meaningful correlations or relationships between performance during PavCA with either CCP or CCC.

We did not measure sensitization of cocaine-induced headwaving during CCP in the UBuff cohort. Repeated i.p. cocaine injections can produce a sensitized headbobbing response in rats ${ }^{38}$, and rats from the UBuff cohort exhibiting reduced locomotor activation after repeated cocaine exposure could be a result of either tolerance to the locomotor activating effect, or sensitization to cocaine-induced stereotypy. The UMich cohort showed increased head-waving responses at the expense of forward locomotion at the $20 \mathrm{mg} / \mathrm{kg}$ dose, ostensibly indicating that this locomotor domain was not captured during CCP. However, headwaving was not related to tendency to sign- or goal-track during CCC, which ultimately supports the notion that these traits remain largely independent of one another in HS rats. Both cohorts were quite different in age and testing experience, so caution should be used in directly comparing one cohort to the other.

Previously, Pavlovian conditioned approach has been associated with performance on variety of other traits, including drug-conditioning ${ }^{1,2,18,19,39-41}$ and non-drug behaviors ${ }^{21,22}$. At least in HS rats, the relationship between tendency to sign-track and a subset of these other traits may be dissociable. In support of this notion, it has recently been demonstrated that PavCA performance is independent of sensation- and novelty-seeking in a large sample of HS rats ${ }^{30}$, suggesting drug-related traits can be dissociated in a sufficiently diverse and large subject pool. This is the first instance in which the HS strain been used extensively for cocaine conditioning in relation to PavCA, and independence of these particular traits may reflect genotypic and phenotypic diversity unique to the HS population that is not present in other commonly used strains such as Sprague-Dawley rats. Not every behavioral task was independent from each other, as PavCA was related to CRF, suggesting that there is a fundamental dissociation between the processes underlying attribution of incentive salience to reward cues, CCP, and CCC within HS rats. Our PCA factor loadings further support this finding, in that measures of incentive salience (terminal index and lever-directed behavior during CRF) showed independent loadings from conditioned and unconditioned locomotor activation in both cohorts of rats. Although other populations of rats may indeed show a different degree of relatedness between these particular tasks, HS rats in particular would be useful in examining each of these particular traits in isolation of the other. Behavioral heterogeneity has been linked to strain and genetic lineage in rodents for Pavlovian conditioned approach ${ }^{42-44}$ and locomotor sensitivity to cocaine $\mathrm{e}^{45,46}$, and thus we suggest HS rats may be a well suited tool for genetic mapping within specific behavioral domains.

In addition, there were pronounced sex differences across each of the tests employed here, particularly during PavCA, CRF, and the locomotor effects of cocaine in CCP and CCC. While we are not the first to report sex differences during PavCA ${ }^{21,30,47,48}$, cocaine-induced locomotion ${ }^{49,50}$, or place preference ${ }^{51}$, we have replicated a variety of previous reports on these differences using a large sample size. In light that these traits are arguably independent, the replication of previously identified sex differences reinforces the need to examine both males and females in the behavioral research, with particular attention to how the biological etiology of behavior differs between the two sexes. The HS line of rats in particular may be a useful tool specifically for examining the genetic and etiological basis of sex differences within a specific behavior of interest, while being able to dissociate it from other previously linked traits.

Characterizing the genetic, epigenetic, transcriptomic, and neurobiological differences between subjects will be crucial for explaining the heterogeneity of behavioral responses to food and drug rewards and cues. Future work will entail examining genome-wide associations between behavior and genetic loci. However, a variety of other mechanisms also warrant consideration. Epigenetic factors, including chromatin-remodeling and modifying mechanisms ${ }^{52}$, deserve attention given their role in learning and memory ${ }^{53}$ and drug-induced changes in plasticity and behavior ${ }^{54}$. Epigenetic changes that occur as a consequence of experience and drug-exposure likely affects individuals differently depending on their genetic landscape. These effects are likely relevant at the neuroanatomical level for any given behavioral trait. These mechanisms may, for example, explain differences in sensitization to the locomotor activating effect of cocaine, including identified mechanisms such as histone ${ }^{55}$ and chromatin ${ }^{56}$ modifications in the nucleus accumbens (NAcc). In addition to the NAcc, the prefrontal cortex (PFC) receives significant monoaminergic innervation, including midbrain dopamine ${ }^{57}$, is critical for learning and memory ${ }^{58}$, and is implicated in the expression of sign-tracking ${ }^{59}$ and responses to cocaine-related stimuli ${ }^{60}$. Genetic and transcriptomic modifications in the PFC are potential substrates that may underlie differences in the motivational properties of food and drug cues, possibly by altering neurobiological function through various pathways such as BDNF ${ }^{61}$.

This study is the first to examine unconditioned and conditioned cocaine responses in relation to Pavlovian conditioned approach with a large sample size. Most of the behavioral measures in both tasks were largely 
unrelated, and those related traits that were identified showed marginal contribution to the unconditioned effects of cocaine. These data underscore the importance of conceptualizing addiction more generally as a multifaceted process, in which multiple independent traits and pathways may result in maladaptive drug use behavior. Further, this work suggests that although these traits may be similar in other strains, caution should be used in interpreting results across studies using different subjects and sample sizes.

\section{Materials and methods}

Subjects. NMcwi:HS (here after referred to as HS) rats were shipped from the laboratory of Dr. Leah Solberg-Woods at Wake Forest University School of Medicine to either the Research Institute on Addiction (RIA) at the University at Buffalo (UBuff; $n=1528$ ) or the Department of Psychology at the University of Michigan (UMich; $n=1188$ ) at approximately 33 days old, as part of the NIDA Center for GWAS in Outbred Rats. These HS rats were established at the NIH from eight founder strains of separate lineages ${ }^{62}$, and are maintained using 64 breeding pairs using a breeding scheme that accounts for kinship coefficients to minimize inbreeding and maintain genetic heterogeneity. They show high genotypic and phenotypic diversity, and are useful for the complex mapping of genetic correlates for a variety of behaviors ${ }^{26,43,63}$.

Rats of the same sex were pair-housed (UBuff) or triple-housed (UMich) in plastic cages $(42.5 \times 22.5 \times 19.25 \mathrm{~cm})$. Cages were lined with bedding (Aspen Shavings) and kept in a temperature-controlled environment $\left(22 \pm 1{ }^{\circ} \mathrm{C}\right)$. No environmental enrichment was provided throughout the experiment. Water and food (Harlan Teklad Laboratory Diet \#8604, Harlan Inc., Indianapolis, IN, USA) were freely available. Following the completion of the RIA phase of testing (see next paragraph), rats were same-day shipped from the RIA to UBuff by the University at Buffalo's laboratory animal facility staff (25-min commute). It is established that exposure to light is sufficient to phase shift the circadian $\operatorname{clock}^{64,65}$. To allow rats to reacclimate and allow sufficient adaptation to photic re-entrainment, rats were given a minimum of 7 days following transfer to adjust before testing began. Rats were handled daily upon arrival to the UBuff testing site. Housing was maintained on the same $12 \mathrm{~h}$ reverse light/dark cycle (lights off at $0730 \mathrm{~h}$ ) at both the RIA and UBuff testing sites, such that they only experienced light outside their typical cycle during transit. Daily testing began during the dark phase $1 \mathrm{~h}$ following lights off. Subjects were tested as groups in the same order daily, such that each subject began testing at the same point in their dark cycle across days. The final group concluded by 5 pm each day, 2 and a half hours prior to lights on. For rats tested at the UMich, behavioral testing began at approximately 60 days old. Rats were then tested for Pavlovian conditioned approach, "novelty-seeking", "sensation-seeking", and cocaine contextual seeking (CCC) as described below and in Table 1.

For rats tested at the UBuff, rats first arrived at the Laboratory Animal Facility and were kept in quarantine for 14 days. They were then transferred to the Research Institute on Addictions in Buffalo, NY, and tested in several paradigms at age PND72. These paradigms, the data from which are the subject of a separate publication, included locomotor activity, light reinforcement, choice reaction time task, delay discounting, and social reinforcement. At the beginning of testing, the average weight of females was $197 \mathrm{~g}$, and the average weight of males was $315 \mathrm{~g}$. The rats were then transferred to the Psychology department and began testing (mean PND162, range 140-204) for Pavlovian Conditioned Approach (PavCA) and Cocaine Cue Preference (CCP) as described below and in Table 1. Rats were tested in 16 batches, each batch consisted of 7 groups of 16 subjects. Rats were tested in the same order daily. All studies were conducted according to the National Research Council (2003) "Guidelines for the Care and Use of Mammals in Neuroscience and Behavioral Research". All procedures were approved by the University at Buffalo and University of Michigan Institutional Animal Care and Use Committees.

Drugs. During CCP and CCC, subjects were treated with either $0.9 \%$ physiological saline, or 10 and $15 \mathrm{mg} /$ $\mathrm{kg}$ injections (i.p.) of cocaine $\mathrm{HCl}$ (Nat. Inst. Of Drug Abuse, Bethesda, MD) dissolved into sterile saline at 10 or $15 \mathrm{mg} / \mathrm{mL}$ respectively. All injections were given immediately prior to conditioning sessions.

Apparatus. Pavlovian conditioned approach (PavCA). Testing occurred in 16 modular testing chambers $(20.5 \times 24.1 \mathrm{~cm}$ floor area, $29.2 \mathrm{~cm}$ high; MED-Associates Inc., St. Albans, VT) located inside either Med-Associates (UMich) or custom-built (UBuff) sound and light attenuating chambers equipped with fans for ventilation and noise masking (A\&B Display Systems, Bay City, MI). $45 \mathrm{mg}$ banana pellets were delivered via a pellet dispenser into a food cup equipped with an infrared photobeam detector to detect head entries. Each chamber contained a retractable backlit lever $(2 \mathrm{~cm}$ length, $6 \mathrm{~cm}$ above floor) on either the left or right side of the food cup. A red houselight was located on top of opposing wall of the chamber $(27 \mathrm{~cm} \mathrm{high})$. During the conditioned reinforcement test, the retractable lever was moved to the center of the wall and the food-cup was removed. Two nosepoke ports with head-entry detectors were situated on the left and right side of the lever. All data were collected using the Med-PC IV software package.

Cocaine conditioned cue preference (CCP). Rats were tested in the dark in black acrylic chambers $(47 \mathrm{~cm}$ length $\times 19 \mathrm{~cm}$ width $\times 30 \mathrm{~cm}$ height) with either "grid" or "hole" textured floors that were spray painted black. Beneath the textured floors was an additional smooth black matte floor. During testing, subjects were video recorded using infrared cameras connected to a 16-channel DVR (Swann Communications, Inc., Santa Fe Springs, CA) to analyze locomotor activity and side/floor preference. Videos were analyzed in real-time using Topscan video tracking software (Clever Sys. Inc., Reston, VA) ${ }^{3,27}$. All testing environments were located in custom-built light- and sound-attenuating chambers.

Cocaine contextual conditioning (CCC). Rats were tested in chambers composed of an outer box (27 in length $\times 13$ in width $\times 26$ in height) and a smaller, insert box (18 in length $\times 6$ in width $\times 22$ in height) that was 
placed in the center of the outer box. Both the outer box and insert were composed of 4 fiberboard walls and did not include a floor or ceiling. A wire mesh was suspended within the outer box, two inches up from the bottom. The mesh support and the inside of the insert box were painted matte grey with Rust-Oleum automobile primer in order to provide the best contrast for the various colors of rats. Each session of the CCC procedure was video recorded with a Zmodo, ZMD-DR SFN6 DVR and analyzed using Noldus Ethovision motion tracking software.

Procedure. Pavlovian conditioned approach (PavCA). During PavCA, subjects learned the association between presentation of a banana-flavored food pellet and a backlit lever-CS over 5 sessions. In the two days prior to testing, subjects received home cage exposure to banana flavored food pellets $(\sim 25$ pellets per day; Bio-Serv, Flemington, NJ, \#F0059). Rats then received a single day of food cup training to habituate subjects to the testing environment. During food-cup training, subjects underwent a 5-min chamber habituation period during which the houselight was extinguished. Next, the houselight was illuminated, and subjects received 25 pellets delivered into the food-cup on a VI-30 s (1-60 s range) schedule. The session ended after the 25 pellets were delivered.

Next, over five daily conditioning sessions, there were 25 lever-food pairings such that delivery of each pellet into the food-cup was preceded by insertion of the lever for 8-s. Lever presses had no programmed consequences. Intervals between trials were determined using a VI-90 schedule (30-150 s range) such that sessions lasted an average of $37.5 \mathrm{~min}$.

Measures. Across the five conditioning sessions, two conditioned responses were measured during the presentation of the lever-CS: lever-directed approach (number of lever deflections) and goal-directed approach (entries into the food cup). Approach latency and food cup entries during the inter-trial interval (outside of the CS period) were also collected.

Previously, we have used these measures to calculate a PavCA index; the general tendency to approach either the lever ("sign-tracking") or food-cup ("goal-tracking") ${ }^{15}$. The index is computed by first measuring: (1) The probability differential of contact with the lever versus food-cup during each CS period (average probability of a lever press on a given CS trial-average probability of a food-cup entry on a given CS trial), (2) the response bias directed towards either the lever or the food cup (\# lever contacts-\# food-cup contacts / \# lever + \# foodcup contacts), and finally (3) the average latency across trials to initiate contact with either the lever or food-cup (food-cup latency - lever latency / 8). These three measures were averaged together, producing an overall PavCA index between -1 and 1, used to categorize subjects as sign-trackers (STs) and goal-trackers (GTs) based on the average index from sessions 4 and 5 of training ${ }^{15}$. Finally, subjects were classed as goal-trackers if their score was between -1 and -0.5 , as intermediates (IN) between -0.5 and 0.5 , and as sign-trackers between 0.5 and +1 .

Our reasoning for using the index as our primary measure of comparison is because it considers all six measures of tendency to sign- and goal- track, and thus allowed us to use a singular measure to categorize subjects into intermediate, sign-, and goal-tracker phenotypes for the purpose of group comparisons. However, although index is useful for categorizing subjects and presenting them a singular distribution, it quashes individual variability for each measure between subjects, even in the same range of the index, based on tendency of overall behavior. Thus, complexities such as the magnitude of sign- and goal-tracking are lost. For example, even within sign-trackers, raw number of lever presses for the food-predictive lever is highly variable across subjects.

To examine whether the distribution of subjects was artifactually different between sexes, we compared raw measures of lever and food-cup directed behavior using lever deflections and food cup entries during the lever CS period. It is worth noting that the pattern of results shown in Fig. 2 presented here also fit the pattern of results from Fig. 2 described in Hughson et al. ${ }^{30}$. We found that females showed a modest positive skew in lever deflections and also in particular food-cup entries (Supplementary Fig. 1a,b), supporting the notion that the bimodality of the index is also reflected by both heightened sign- and goal-tracking in the upper quartile range for both behaviors. Similarly, most males showed a positive skew for lever deflections, driven by the limited number of sign-trackers in the overall distribution, but showed a more normal distribution for goal-tracking (Supplementary Fig. 1a,b) suggesting the index works as a partial surrogate, at least for these two measures. To ensure that the reported correlations between tasks weren't contaminated by use of the index, we also examined the relationship between lever contacts and food-cup entries with three major measures described. Here, we found that, although several correlations between lever contacts, food cup entries, and performance during conditioned cue preference were significant, they all yielded relatively small $r^{2}$ values (Supplementary Fig. 1c), with the largest being $\mathrm{r}^{2}=0.026$, thus supporting our original conclusion that performance on one task is largely unrelated to the other, even when using measures other than the index.

Conditioned reinforcement (CRF) test and measures. The ability of the food-associated lever-CS to reinforce the acquisition of a new instrumental response (nosepoking) was assessed the day after Pavlovian conditioning ended. Testing occurred in the same chamber used for PavCA but the center food-cup was removed and replaced with the illuminated backlit lever-CS. On both the left and the right side of the lever-CS were two nosepoke ports, one active and one inactive. All other aspects of the testing environment were identical. Nosepokes into the active hole resulted in insertion of the lever-CS into the chamber for $3 \mathrm{~s}$, during which lever deflections were recorded. Nosepokes into the inactive port had no programmed consequences. Sessions lasted $40 \mathrm{~min}$. The primary measures were entries into active and inactive ports, lever deflections, and number of earned lever presentations. We chose to separately examine nosepokes from earned lever presentations and lever deflections with the idea that responses into a novel nosepoke port and lever-directed responses might differ in terms of their relationship to PavCA Phenotype ${ }^{30}$. We ultimately measured lever-directed behavior by using lever presses made per earned reinforcer, a similar outcome measure used in the UMich cohort, the Incentive Value Index ((responses in active port - responses in inactive port) + number of lever presses). 
Cocaine conditioned cue preference (CCP). During CCP, rats learned the association between a textured floor stimulus and a $10 \mathrm{mg} / \mathrm{kg}$ injection of cocaine. Throughout the 11 days of testing, rats were weighed daily and placed into individual transport containers (Sterilite Corporation, Townsend, MA), for 15 min before being moved into the testing room. Subjects first received one day of habituation in which subjects were injected with saline and then placed in the testing environment with a smooth matte floor for $30 \mathrm{~min}$ to allow rats to acclimate to the chamber. On the following day, subjects received a saline injection and underwent a 30 min "pre-test" in which the testing chamber was outfitted with both the "hole" and "grid" floor halves, counterbalanced for left/ right position. Subjects were counter-conditioned, such that the least preferred floor (the floor each subject spent the least amount of time on) was assigned as the cocaine-paired floor. On the following 8 days, subjects received $10 \mathrm{mg} / \mathrm{kg}$ cocaine and i.p. saline injections (order counterbalanced) on alternating days before being placed in the chamber containing a single floor type. Each pair of cocaine and saline conditioning sessions was termed a trial, for a total of 4 trials. Finally, subjects received a post-test, in which they were tested in the presence of both floors following a saline injection. The time spent on the cocaine-paired floor was measured in seconds on both the pre- and post-test sessions. The change in time spent on the cocaine floor was determined by subtracting post-test time from pre-test time. Distance travelled in $\mathrm{mm}$ across all testing days was determined by using Topscan's locomotor analysis.

Cocaine contextual conditioning (CCC). During CCC, rats learned to associate a context with a $15 \mathrm{mg} / \mathrm{kg}$ cocaine injection. Importantly, CCC differed from CCP in that cocaine pairings occurred with the entire testing context, rather than with exchangeable tactile floor stimuli. First, subjects underwent a single 30-min session of exposure to the testing apparatus with no prior injection to measure locomotor response to novelty (Day 1 ). On the next day (Day 2), subjects received an injection of saline and underwent an additional 30-min pre-conditioning session. Subjects then began cocaine contextual conditioning (Days 3-7) in which rats were treated with cocaine immediately prior to each test session. Finally, on the last day (Day 8) subjects received a 30-min postconditioning test session following an injection of saline, to assess the degree of context conditioned hyperactivity (adapted from: ${ }^{66}$ ). Throughout testing, two measures of locomotor activity were computer scored: overall distance travelled, and bouts of head waving as described in ${ }^{27}$. Acute locomotor activation by cocaine was analyzed by comparing Day 3-Day 2. Locomotor sensitization to cocaine was analyzed by comparing Day 3-Day 7. The conditioned locomotor response to the cocaine-paired environment was analyzed by comparing Day 2-Day 8.

Analyses. Repeated-measures analysis of variance (ANOVA), in conjunction with Tukey's HSD post-hoc tests, were used to probe significant main effects and interactions. For PavCA, CRF, cocaine CCP, and CCC, Sex (male, female), and PavCAPheno (ST, IN, GT) were the between-subjects factors. For PavCA, Session (1-5) was the within-subjects repeated measures factor. For CRF, Port (active, inactive) was the within-subjects repeated measures factor. Lever-directed behavior during CRF (lever presses, lever presses per reinforcer) were both analyzed separately from nosepoking behavior. For cocaine CCP, conditioning Trial (1-4), Test (pre, post) and Drug (saline, cocaine) were the within-subjects repeated measures factors. For CCC, Trial $(1,5)$ and Test (Pre, Post) were the within-subjects repeated measures factors. For CCC, of the 1188 phenotyped subjects, 15 were dropped due to data collection error and were casewise excluded from all CCC analyses. Further note that the rats presented in the CCC experiment were used in a separate publication examining the relationship between PavCA, response to novelty, and sensation seeking ${ }^{30}$. For brevity, we do not present a dedicated results section for this particular batch of Pavlovian conditioned approach, because the results of these data are similar to the University at Buffalo cohort, and have been described in detail previously ${ }^{30}$.

Further, because subjects in the UBuff cohort arrived at different ages, we ran all analyses presented here using age at the start of testing as a continuous predictor for each variable. While several dependent variables (food cup CS entries, food cup CS entry probability, food cup CS latency, PavCA index, port responses during CRF, earned reinforcers during CRF, change in time on cocaine CS +, CCP locomotor activity, conditioned locomotion) yielded significant main effects or interactions with age at the start of testing, the effect size of these findings were extremely small. Locomotor activity and conditioned locomotion during CCP had the largest effects of age $\left(\eta^{2}=0.054,0.015\right)$, with all other measures yielding $\eta^{2}$ below 0.005 . We therefore excluded age as a factor from the primary findings.

In addition, to determine whether the traits discussed here could be reduced to fewer dimensions, two iterations of principal components analysis were conducted in both populations of animals. Specifically, each analysis included index during Pavlovian conditioned approach, lever directed behavior during CRF (lever presses per reinforcer, incentive value index), the first and last days of locomotor activation during CCP and CCC, and the conditioned approach and conditioned locomotion to the cocaine paired floor and context, respectively. All factors examined were determined with a minimum eigenvalue of 1 , and were factor rotated using normalized Varimax.

All statistics for all experiments were computed using Statistica 13 (Dell Inc., Tulsa, OK). Box and jitter plots were constructed in R (R version 3.6.1., R Studio, Boston, MA) using the ggplot2 package. Principal components plots were generated in Statistica 13. All plots both were modified to improve visual clarity using Adobe Illustrator 2020 (Adobe, San Jose, CA).

Dual publication statement. The preprint for this submission is available online at BioRxiv. In addition, the rats used in the University of Michigan cohort are also part of a separate publication Hughson et al. ${ }^{30}$. In this publication, rats were compared on the Pavlovian conditioned approach task with two measures of noveltyseeking and sensation-seeking. None of the data from that publication appear directly in this submission. However, their Pavlovian conditioned approach data was used for comparison with a new procedure reported here, 
cocaine contextual conditioning (CCC). The primary findings, results, and conclusions presented in this paper address a different scientific question than those presented in Hughson et al. ${ }^{30}$.

\section{Data availability}

The datasets generated and/or analyzed during the current study are available on our project webpage (ratgenes. org) or from the corresponding author on reasonable request.

Received: 18 May 2020; Accepted: 15 December 2020

Published online: 26 January 2021

\section{References}

1. Saunders, B. T. \& Robinson, T. E. A cocaine cue acts as an incentive stimulus in some but not others: implications for addiction. Biol. Psychiatry 67, 730-736. https://doi.org/10.1016/j.biopsych.2009.11.015 (2010).

2. Versaggi, C. L., King, C. P. \& Meyer, P. J. The tendency to sign-track predicts cue-induced reinstatement during nicotine selfadministration, and is enhanced by nicotine but not ethanol. Psychopharmacology 233, 2985-2997. https://doi.org/10.1007/s0021 3-016-4341-7 (2016).

3. Meyer, P. J., Ma, S. T. \& Robinson, T. E. A cocaine cue is more preferred and evokes more frequency-modulated $50-\mathrm{kHz}$ ultrasonic vocalizations in rats prone to attribute incentive salience to a food cue. Psychopharmacology 219, 999-1009. https://doi.org/10.1007/ s00213-011-2429-7 (2012).

4. Molander, A. C. et al. High impulsivity predicting vulnerability to cocaine addiction in rats: some relationship with novelty preference but not novelty reactivity, anxiety or stress. Psychopharmacology 215, 721-731. https://doi.org/10.1007/s00213-011-2167-x (2011).

5. Beckmann, J. S., Marusich, J. A., Gipson, C. D. \& Bardo, M. T. Novelty seeking, incentive salience and acquisition of cocaine selfadministration in the rat. Behav. Brain Res. 216, 159-165. https://doi.org/10.1016/j.bbr.2010.07.022 (2011).

6. Belin, D., Berson, N., Balado, E., Piazza, P. V. \& Deroche-Gamonet, V. High-novelty-preference rats are predisposed to compulsive cocaine self-administration. Neuropsychopharmacology 36, 569-579. https://doi.org/10.1038/npp.2010.188 (2011).

7. Piazza, P. V., Deminiere, J. M., Le Moal, M. \& Simon, H. Factors that predict individual vulnerability to amphetamine selfadministration. Science 245, 1511-1513. https://doi.org/10.1126/science.2781295 (1989).

8. Gancarz, A. M., Robble, M. A., Kausch, M. A., Lloyd, D. R. \& Richards, J. B. Sensory reinforcement as a predictor of cocaine and water self-administration in rats. Psychopharmacology 226, 335-346. https://doi.org/10.1007/s00213-012-2907-6 (2013).

9. Gancarz, A. M., Robble, M. A., Kausch, M. A., Lloyd, D. R. \& Richards, J. B. Association between locomotor response to novelty and light reinforcement: sensory reinforcement as a rodent model of sensation seeking. Behav. Brain Res. 230, 380-388. https:// doi.org/10.1016/j.bbr.2012.02.028 (2012).

10. Belin, D., Mar, A. C., Dalley, J. W., Robbins, T. W. \& Everitt, B. J. High impulsivity predicts the switch to compulsive cocaine-taking. Science 320, 1352-1355. https://doi.org/10.1126/science.1158136 (2008).

11. de Wit, H. Impulsivity as a determinant and consequence of drug use: a review of underlying processes. Addict Biol. 14, 22-31. https://doi.org/10.1111/j.1369-1600.2008.00129.x (2009).

12. Economidou, D., Pelloux, Y., Robbins, T. W., Dalley, J. W. \& Everitt, B. J. High impulsivity predicts relapse to cocaine-seeking after punishment-induced abstinence. Biol. Psychiatry 65, 851-856. https://doi.org/10.1016/j.biopsych.2008.12.008 (2009).

13. Perry, J. L., Larson, E. B., German, J. P., Madden, G. J. \& Carroll, M. E. Impulsivity (delay discounting) as a predictor of acquisition of IV cocaine self-administration in female rats. Psychopharmacology 178, 193-201. https://doi.org/10.1007/s00213-004-1994-4 (2005).

14. Hearst, E. \& Jenkins, H. M. Sign-Tracking: The Stimulus-Reinforcer Relation and Directed Action (Psychonomic Society, Chicago, 1974).

15. Meyer, P. J. et al. Quantifying individual variation in the propensity to attribute incentive salience to reward cues. PLoS ONE 7, e38987. https://doi.org/10.1371/journal.pone.0038987 (2012).

16. Boakes, R. A. Performance on learning to associate a stimulus with positive reinforcement. Operant-Pavlovian Interact. 67-97 (1977).

17. Robinson, T. E., Carr, C. C. \& Kawa, A. B. The propensity to attribute incentive salience to drug cues and poor cognitive control combine to render sign-trackers susceptible to addiction. Sign-tracking and drug addiction (Vol. A). 10 (Maize Books, Ann Arbor, MI, 2018).

18. Tripi, J. A., Dent, M. L. \& Meyer, P. J. Individual differences in food cue responsivity are associated with acute and repeated cocaineinduced vocalizations, but not cue-induced vocalizations. Psychopharmacology 234, 437-446 (2017).

19. Saunders, B. T. \& Robinson, T. E. Individual variation in the motivational properties of cocaine. Neuropsychopharmacology 36, 1668-1676. https://doi.org/10.1038/npp.2011.48 (2011).

20. Pitchers, K. K., Sarter, M. \& Robinson, T. E. The hot "n" cold of cue-induced drug relapse. Learn Mem. 25, 474-480. https://doi. org/10.1101/lm.046995.117 (2018).

21. King, C. P. et al. Premature responding is associated with approach to a food cue in male and female heterogeneous stock rats. Psychopharmacology 233, 2593-2605. https://doi.org/10.1007/s00213-016-4306-x (2016).

22. Lovic, V., Saunders, B. T., Yager, L. M. \& Robinson, T. E. Rats prone to attribute incentive salience to reward cues are also prone to impulsive action. Behav. Brain Res. 223, 255-261. https://doi.org/10.1016/j.bbr.2011.04.006 (2011).

23. Paolone, G., Angelakos, C. C., Meyer, P. J., Robinson, T. E. \& Sarter, M. Cholinergic control over attention in rats prone to attribute incentive salience to reward cues. J. Neurosci. 33, 8321-8335. https://doi.org/10.1523/JNEUROSCI.0709-13.2013 (2013).

24. Hansen, C. \& Spuhler, K. Development of the National Institutes of Health genetically heterogeneous rat stock. Alcohol. Clin. Exper. Res. 8, 477-479 (1984).

25. Spuhler, K. \& Deitrich, R. A. Correlative analysis of ethanol-related phenotypes in rat inbred strains. Alcohol. Clin. Exp. Res. 8, 480-484. https://doi.org/10.1111/j.1530-0277.1984.tb05707.x (1984).

26. Solberg Woods, L. C. \& Palmer, A. A. in Rat Genomics (eds G. Thomas Hayman, Jennifer R. Smith, Melinda R. Dwinell, \& Mary Shimoyama) 233-247 (Springer, New York, 2019).

27. Flagel, S. B. \& Robinson, T. E. Quantifying the psychomotor activating effects of cocaine in the rat. Behav. Pharmacol. 18, 297-302. https://doi.org/10.1097/FBP.0b013e3281f522a4 (2007).

28. Cunningham, C. L., Gremel, C. M. \& Groblewski, P. A. Drug-induced conditioned place preference and aversion in mice. Nat. Protoc. 1, 1662-1670. https://doi.org/10.1038/nprot.2006.279 (2006).

29. Vezina, P. \& Stewart, J. Conditioned locomotion and place preference elicited by tactile cues paired exclusively with morphine in an open field. Psychopharmacology 91, 375-380. https://doi.org/10.1007/BF00518195 (1987).

30. Hughson, A. R. et al. Incentive salience attribution, "sensation-seeking" and "novelty-seeking" are independent traits in a large sample of male and female heterogeneous stock rats. Sci. Rep. 9, 2351. https://doi.org/10.1038/s41598-019-39519-1 (2019). 
31. Robinson, T. E. \& Flagel, S. B. Dissociating the predictive and incentive motivational properties of reward-related cues through the study of individual differences. Biol. Psychiatry 65, 869-873. https://doi.org/10.1016/j.biopsych.2008.09.006 (2009).

32. Antoniou, K., Kafetzopoulos, E., Papadopoulou-Daifoti, Z., Hyphantis, T. \& Marselos, M. D-amphetamine, cocaine and caffeine: a comparative study of acute effects on locomotor activity and behavioural patterns in rats. Neurosci. Biobehav. Rev. 23, 189-196. https://doi.org/10.1016/s0149-7634(98)00020-7 (1998).

33. O’Dell, L. E., Khroyan, T. V. \& Neisewander, J. L. Dose-dependent characterization of the rewarding and stimulant properties of cocaine following intraperitoneal and intravenous administration in rats. Psychopharmacology 123, 144-153. https://doi. org/10.1007/BF02246171 (1996).

34. Flagel, S. B., Akil, H. \& Robinson, T. E. Individual differences in the attribution of incentive salience to reward-related cues: Implications for addiction. Neuropharmacology 56(Suppl 1), 139-148. https://doi.org/10.1016/j.neuropharm.2008.06.027 (2009).

35. Flagel, S. B., Watson, S. J., Akil, H. \& Robinson, T. E. Individual differences in the attribution of incentive salience to a rewardrelated cue: influence on cocaine sensitization. Behav. Brain Res. 186, 48-56. https://doi.org/10.1016/j.bbr.2007.07.022 (2008).

36. Flagel, S. B., Waselus, M., Clinton, S. M., Watson, S. J. \& Akil, H. Antecedents and consequences of drug abuse in rats selectively bred for high and low response to novelty. Neuropharmacology 76(Pt B), 425-436. https://doi.org/10.1016/j.neuropharm.2013.04.033 (2014).

37. Flagel, S. B. et al. An animal model of genetic vulnerability to behavioral disinhibition and responsiveness to reward-related cues: implications for addiction. Neuropsychopharmacology 35, 388-400. https://doi.org/10.1038/npp.2009.142 (2010).

38. O'Dell, L. E., Khroyan, T. V. \& Neisewander, J. L. Dose-dependent characterization of the rewarding and stimulant properties of cocaine following intraperitoneal and intravenous administration in rats. Psychopharmacology 123, 144-153. https://doi. org/10.1007/BF02246171 (1996).

39. Villaruel, F. R. \& Chaudhri, N. Individual differences in the attribution of incentive salience to a pavlovian alcohol cue. Front. Behav. Neurosci. 10, 238. https://doi.org/10.3389/fnbeh.2016.00238 (2016).

40. Yager, L. M., Pitchers, K. K., Flagel, S. B. \& Robinson, T. E. Individual variation in the motivational and neurobiological effects of an opioid cue. Neuropsychopharmacology 40, 1269-1277. https://doi.org/10.1038/npp.2014.314 (2015).

41. Yager, L. M. \& Robinson, T. E. A classically conditioned cocaine cue acquires greater control over motivated behavior in rats prone to attribute incentive salience to a food cue. Psychopharmacology 226, 217-228. https://doi.org/10.1007/s00213-012-2890-y (2013).

42. Dickson, P. E. et al. Sex and strain influence attribution of incentive salience to reward cues in mice. Behav. Brain. Res. 292, 305-315. https://doi.org/10.1016/j.bbr.2015.05.039 (2015).

43. Parker, C. C. et al. Rats are the smart choice: rationale for a renewed focus on rats in behavioral genetics. Neuropharmacology 76(Pt B), 250-258. https://doi.org/10.1016/j.neuropharm.2013.05.047 (2014).

44. Fitzpatrick, C. J. et al. Variation in the form of Pavlovian conditioned approach behavior among outbred male Sprague-Dawley rats from different vendors and colonies: sign-tracking vs. goal-tracking. PLoS ONE 8, e75042. https://doi.org/10.1371/journ al.pone.0075042 (2013).

45. Saul, M. C. et al. Genetic variation and sex differences are missed opportunities for addiction biology. bioRxiv https://doi. org/10.1101/2020.02.14.949784 (2020).

46. Schoenrock, S. A. et al. Characterization of genetically complex collaborative cross mouse strains that model divergent locomotor activating and reinforcing properties of cocaine. Psychopharmacology 237, 979-996. https://doi.org/10.1007/s00213-019-05429-3 (2020).

47. Peterson, V. L. et al. Sex, drugs, and the microbiome: goal/sign-tracking phenotype reveals associations between behavior and microbiome in a sex-dependent manner in the rat (2018).

48. Pitchers, K. K. et al. Individual variation in the propensity to attribute incentive salience to a food cue: influence of sex. Behav. Brain Res. 278, 462-469. https://doi.org/10.1016/j.bbr.2014.10.036 (2015).

49. van Haaren, F. \& Meyer, M. E. Sex differences in locomotor activity after acute and chronic cocaine administration. Pharmacol. Biochem. Behav. 39, 923-927. https://doi.org/10.1016/0091-3057(91)90054-6 (1991).

50. Festa, E. D. et al. Sex differences in cocaine-induced behavioral responses, pharmacokinetics, and monoamine levels. Neuropharmacology 46, 672-687. https://doi.org/10.1016/j.neuropharm.2003.11.017 (2004).

51. Russo, S. J. et al. Sex differences in the conditioned rewarding effects of cocaine. Brain Res. 970, 214-220. https://doi.org/10.1016/ s0006-8993(03)02346-1 (2003).

52. McQuown, S. C. \& Wood, M. A. Epigenetic regulation in substance use disorders. Curr. Psychiatry Rep. 12, 145-153. https://doi. org/10.1007/s11920-010-0099-5 (2010).

53. Dias, B. G., Maddox, S., Klengel, T. \& Ressler, K. J. Epigenetic mechanisms underlying learning and the inheritance of learned behaviors. Trends Neurosci. 38, 96-107. https://doi.org/10.1016/j.tins.2014.12.003 (2015).

54. Nestler, E. J. Epigenetic mechanisms of drug addiction. Neuropharmacology 76(Pt B), 259-268. https://doi.org/10.1016/j.neuro pharm.2013.04.004 (2014).

55. Heller, E. A. et al. Targeted epigenetic remodeling of the $c \mathrm{dk} 5$ gene in nucleus accumbens regulates cocaine- and stress-evoked behavior. J. Neurosci. 36, 4690-4697. https://doi.org/10.1523/JNEUROSCI.0013-16.2016 (2016).

56. Damez-Werno, D. et al. Drug experience epigenetically primes Fosb gene inducibility in rat nucleus accumbens. J. Neurosci. 32, 10267-10272. https://doi.org/10.1523/JNEUROSCI.1290-12.2012 (2012).

57. Floresco, S. B. \& Magyar, O. Mesocortical dopamine modulation of executive functions: beyond working memory. Psychopharmacology 188, 567-585. https://doi.org/10.1007/s00213-006-0404-5 (2006).

58. Robbins, T. W. \& Roberts, A. C. Differential regulation of fronto-executive function by the monoamines and acetylcholine. Cereb. Cortex 17(Suppl 1), i151-160. https://doi.org/10.1093/cercor/bhm066 (2007).

59. Tomie, A., Tirado, A. D., Yu, L. \& Pohorecky, L. A. Pavlovian autoshaping procedures increase plasma corticosterone and levels of norepinephrine and serotonin in prefrontal cortex in rats. Behav. Brain Res. 153, 97-105. https://doi.org/10.1016/j.bbr.2003.11.006 (2004).

60. Franklin, T. R. \& Druhan, J. P. Involvement of the nucleus accumbens and medial prefrontal cortex in the expression of conditioned hyperactivity to a cocaine-associated environment in rats. Neuropsychopharmacology 23, 633-644. https://doi.org/10.1016/S0893 $-133 \mathrm{X}(00) 00162-7$ (2000).

61. Morrow, J. D., Saunders, B. T., Maren, S. \& Robinson, T. E. Sign-tracking to an appetitive cue predicts incubation of conditioned fear in rats. Behav. Brain Res. 276, 59-66. https://doi.org/10.1016/j.bbr.2014.04.002 (2015).

62. Hansen, C. \& Spuhler, K. Development of the National Institutes of Health genetically heterogeneous rat stock. Alcohol. Clin. Exp. Res. 8, 477-479. https://doi.org/10.1111/j.1530-0277.1984.tb05706.x (1984).

63. Mott, R., Talbot, C. J., Turri, M. G., Collins, A. C. \& Flint, J. A method for fine mapping quantitative trait loci in outbred animal stocks. Proc. Natl. Acad. Sci. USA 97, 12649-12654. https://doi.org/10.1073/pnas.230304397 (2000).

64. Pittendrigh, C. S. \& Daan, S. A functional analysis of circadian pacemakers in nocturnal rodents. J. Comp. Physiol. 106, 291-331. https://doi.org/10.1007/bf01417859 (1976).

65. Hughes, S., Jagannath, A., Hankins, M. W., Foster, R. G. \& Peirson, S. N. in Methods in Enzymology Vol. 552 (ed Amita Sehgal) 125-143 (Academic Press, 2015).

66. Beninger, R. J. \& Herz, R. S. Pimozide blocks establishment but not expression of cocaine-produced environment-specific conditioning. Life Sci. 38, 1425-1431. https://doi.org/10.1016/0024-3205(86)90476-5 (1986). 


\section{Author contributions}

C.P.K. collected data from the University at Buffalo cohort, and analyzed and plotted the data shown in all figures, and wrote the submitted manuscript with the input of the other authors. J.A.T. and A.C.L collected, compiled, and analyzed the data from the University at Buffalo cohorts (Figs. 1, 2, 3, 4 and 7). A.R.H. and A.P.H collected, compiled, and analyzed the data from the from the University of Michigan cohort (Figs. 5, 6 and 7). K.L.H and L.C.S.W maintained the heterogeneous stock rats under rotational breeding, and supplied them to the phenotyping cores. A.C., O.P, and A.A.P. generated some of the ideas for the project and analyses, and A.A.P. served as the Principal Investigator for the NIDA Center of Excellence (P50 DA037844) grant which funded the work. K.I., T.E.R., S.B.F., and P.J.M conceived the idea for the project, designed and oversaw the experiments, and edited and revised other portions of the manuscript. All authors read and approved the final manuscript.

\section{Competing interests}

The authors declare no competing interests.

\section{Additional information}

Supplementary Information The online version contains supplementary material available at https://doi. org/10.1038/s41598-020-80798-w.

Correspondence and requests for materials should be addressed to P.J.M.

Reprints and permissions information is available at www.nature.com/reprints.

Publisher's note Springer Nature remains neutral with regard to jurisdictional claims in published maps and institutional affiliations.

(c) (i) Open Access This article is licensed under a Creative Commons Attribution 4.0 International License, which permits use, sharing, adaptation, distribution and reproduction in any medium or format, as long as you give appropriate credit to the original author(s) and the source, provide a link to the Creative Commons licence, and indicate if changes were made. The images or other third party material in this article are included in the article's Creative Commons licence, unless indicated otherwise in a credit line to the material. If material is not included in the article's Creative Commons licence and your intended use is not permitted by statutory regulation or exceeds the permitted use, you will need to obtain permission directly from the copyright holder. To view a copy of this licence, visit http://creativecommons.org/licenses/by/4.0/.

(C) The Author(s) 2021 